\title{
Either IL-7 activation of JAK-STAT or BEZ inhibition of PI3K-AKT-mTOR pathways dominates the single-cell phosphosignature of ex vivo treated pediatric T-cell acute lymphoblastic leukemia cells
}

Daniela Kuzilková, ${ }^{1,2^{\star}}$ Cristina Bugarin, ${ }^{3 *}$ Katerina Rejlova, ${ }^{1,2}$ Axel R. Schulz, ${ }^{4}$ Henrik E. Mei, ${ }^{4}$ Maddalena Paganin, ${ }^{5}$ Alessandra Biffi, ${ }^{5}$ Andrea Biondi, ${ }^{3}$ Tomas Kalina ${ }^{1,2 \#}$ and Giuseppe Gaipa ${ }^{3 \#}$

${ }^{1}$ Childhood Leukemia Investigation Prague, Prague, Czech Republic; ${ }^{2}$ Department of Pediatric Hematology and Oncology, 2nd Faculty of Medicine, Charles University Prague, Prague, Czech Republic; ${ }^{3}$ Fondazione Tettamanti, Clinica Pediatrica Università degli Studi Milano Bicocca, Monza (MB), Italy; ${ }^{4}$ German Rheumatism Research Center Berlin (DRFZ), a Leibniz Institute, Berlin, Germany and ${ }^{5}$ Pediatric Hematology, Oncology and Stem Cell Transplant Division, Women and Child Health Department, University of Padova, Padova, Italy.

*DK and $\mathrm{CB}$ contributed equally as co-first authors.

\#TK and GG contributed equally as co-senior authors.

\author{
Correspondence: \\ Andrea Biondi \\ andrea.biondi@unimib.it \\ Received: $\quad$ March 17, 2021 \\ Accepted: $\quad$ October 12, 2021 \\ Prepublished: October 21, 2021 \\ https://doi.org/10.3324/haematol.2021.278796 \\ ○2022 Ferrata Storti Foundation \\ Haematologica material is published under a \\ CC BY-NC license (c) (1) $\Theta$
}

\begin{abstract}
T-cell acute lymphoblastic leukemia (T-ALL) is an aggressive cancer arising from lymphoblasts of T-cell origin. While TALL accounts for only $15 \%$ of childhood and $25 \%$ of adult ALL, $30 \%$ of patients relapse with a poor outcome. Targeted therapy of resistant and high-risk pediatric T-ALL is therefore urgently needed, together with precision medicine tools allowing the testing of efficacy in patient samples. Furthermore, leukemic cell heterogeneity requires drug response assessment at the single-cell level. Here we used single-cell mass cytometry to study signal transduction pathways such as JAK-STAT, PI3K-AKT-mTOR and MEK-ERK in 16 diagnostic and five relapsed T-ALL primary samples, and investigated the in vitro response of cells to Interleukin-7 (IL-7) and the inhibitor BEZ-235. T-ALL cells showed upregulated activity of the PI3K-AKT-mTOR and MEK-ERK pathways and increased expression of proliferation and translation markers. We found that perturbation induced by the ex vivo administration of either IL-7 or BEZ-235 reveals a high degree of exclusivity with respect to the phospho-protein responsiveness to these agents. Notably, these response signatures were maintained from diagnosis to relapse in individual patients. In conclusion, we demonstrated the power of mass cytometry single-cell profiling of signal transduction pathways in T-ALL. Taking advantage of this advanced approach, we were able to identify distinct clusters with different responsiveness to IL-7 and BEZ-235 that can persist at relapse. Collectively our observations can contribute to a better understanding of the complex signaling network governing T-ALL behavior and its correlation with influence on the response to therapy.
\end{abstract}

\section{Introduction}

The incidence of acute lymphoblastic leukemia (ALL) in children peaks between 2 and 5 years of age, and approximately $15 \%$ of cases are of T-cell origin and lead to T-cell ALL (T-ALL). The survival rates of pediatric T-ALL patients have improved in recent years, approaching a 5-year event-free survival of $70-80 \%{ }^{1}$ However, $25 \%$ of children with T-ALL relapse with a generally more aggressive disease with a very poor outcome. Thus, efforts in the investigation of disease biology that might contribute to the development of more effective and specific treatments are urgently needed. ${ }^{2,3}$ Glucocorticoids (GC) represent central components of T-ALL therapy, and the early response to GC-based therapy is an important predictor of longterm outcome. ${ }^{4}$ Accordingly, in Berlin-Frankfurt-Münsteroriented trials for childhood ALL, patients are stratified based on prednisone (PDN) response following 7 days of PDN monotherapy, and PDN poor responders (PPR) have a significantly worse outcome than PDN good responders (PGR) ${ }^{5}$ indicating that intrinsic differences in GC sensitivity at diagnosis impact the outcome. However, despite the 
consolidated use of GC in ALL clinical management, a comprehensive understanding of the mechanisms underlying GC sensitivity is still lacking. ${ }^{6-8}$

The IL-7R-JAK-STAT5 signaling pathway plays a critical role in T-ALL, contributing to leukemogenesis in vivo ${ }^{9-11}$ or driving disease progression by regulating cell viability and proliferation. ${ }^{12-15}$

Somatic gain-of-function mutations in the specific $I L-7 R \alpha$ (CD127) subunit can occur in roughly $10-12 \%$ of pediatric and adult T-ALL cases, leading to constitutive activation of the receptor. ${ }^{16,17}$ Mutations may also occur in downstream effectors of IL-7/IL-7R-mediated signaling, such as JAK-STAT, PI3K-AKT-mTOR and RAS-MEK-ERK pathways which are found in a large proportion (30-50\%) of T-ALL cases. ${ }^{18-21}$ IL-7R pathway mutations are enriched in subtypes of patients overexpressing HOXA and TLX $X^{17,18,22}$ or with early T-cell precursor ALL (ETP-ALL) phenotype. ${ }^{23,24}$ However mutations in IL-7R, JAK1 and JAK3 were also shown to associate with genetic lesions in WT1, $P R C 2$ or PHF6 epigenetic regulators. ${ }^{25}$ Furthermore, the IL-7R-JAKSTAT5 pathway has been investigated for its role in GC resistance in T-ALL. Li et al. ${ }^{19}$ performed whole genome and targeted exome sequencing in patients with T-ALL and found that mutations in the IL-7R signaling components JAK1 and KRAS correlated with steroid resistance and poor outcome in a subset of T-ALL whereas Meyer et al. ${ }^{26}$ showed that IL-7 mediates an intrinsic and physiologic mechanism of GC resistance in normal thymocyte development that is retained during leukemogenesis in a subset of T-ALL. A large cohort of adult T-ALL cases studied by Kim and collaborators contained a subgroup of patients with IL-7R-pathway mutations with slow-response to remission induction regimen measured at day 8 and week $6 .{ }^{21}$

PI3K-AKT-mTOR is a major signaling pathway implicated in T-ALL malignant transformation promoting several functions including cell survival and proliferation. ${ }^{27}$ The major negative regulator of this pathway is the tumor suppressor lipid phosphatase and tensin homolog (PTEN), which is frequently inactivated in human cancer. ${ }^{28,29}$ PTEN non-sense or frame-shift mutations cluster in exon 7, resulting in a C-terminal truncated protein leading to a decreased or absent PTEN expression and activity ${ }^{30}$ resulting in PI3K-AKT aberrant activation.

Gene expression profiling of T-ALL cases revealed subgroups of T-ALL, each characterized by a specific transcriptional profile and the ectopic expression of one particular transcription factor which could impact the differentiation process of the cells. The most represented subgroup shows ectopic TAL1 expression, whereas other major subgroups show mutual exclusive expression of TLX1, TLX3, HOXA9/10, LMO2, or NKX2-1. ${ }^{31-33}$ Furthermore, the ETP-ALL is characterized by aberrant expression of LYL12; hematopoietic transcription factors such as RUNX1 and ETV 6 are frequently mutated in this genetically heterogeneous subgroup. ${ }^{24}$

In addition to the ectopic expression of transcription factors, which can represent an initiating step in T-ALL development, further mutations are required for the full leukemic transformation. These include mutations leading to dysregulation of the activity of PTEN, WT1, RAS, IL-7R and PHF6 affecting several signal transduction pathways implicated in the development of T-ALL. ${ }^{20}$

Relapsed disease represents one of the most challenging settings in the clinical management of T-ALL. Globally, recent studies have indicated that relapsed blasts can evade therapy by utilizing a variety of biological pathways. ${ }^{34}$ Despite the need for targeting molecules to treat resistant and highrisk pediatric T-ALL, in vitro drug testing may not be predictive of in vivo efficacy due to the complexity of the signaling network and due to the cell heterogeneity within the individual sample. ${ }^{35}$ Single-cell mass cytometry analysis represents a promising tool in the comprehension of signaling networks and their role in the response to drugs. ${ }^{36}$ Currently, mass cytometry allows the measurement of more than 40 parameters per single cell, rendering this approach an ideal method to assay drug candidate mechanisms of action and selectivity to cancer cells. ${ }^{37}$ Although many efforts have been made to characterize the genomic landscape of relapsed TALL, many aspects of leukemia resistance are still to be learned. Thus, the integration of genomic and epigenetic studies with new functional proteomic approaches at the single-cell level is essential to continue identifying potential drug targets or biomarkers. In this work, we aimed to evaluate the functional profiles of T-ALL cells collected from children with T-ALL at diagnosis or at relapse and correlate them with their biological and clinical features, including early response to therapy, by mass cytometry.

\section{Methods}

\section{Primary samples and diagnostic procedures}

This study was approved by the Institutional Review Board of San Gerardo Hospital (Monza, Italy), informed consent was obtained from all patients and their guardians in accordance with the Declaration of Helsinki.

Bone marrow (BM) or peripheral blood (PB) mononuclear cells (MNC) were collected from 16 children with T-ALL enrolled in the AIEOP-ALL-2009 or R2006 protocols at the Pediatric Clinic of University Milano Bicocca at San Gerardo Hospital, stored and thawed as described previously. ${ }^{38} \mathrm{Im}$ munophenotyping and evaluation of genetic aberrations were performed as described previously, ${ }^{38}$ and the T-ALL diagnosis was based on consensus criteria. ${ }^{39}$ We also collected PBMNC from three healthy subjects. The immunological classification as well as the clinical and genetic features are reported in Table 1. 


\section{In vitro prednisolone response assay}

In order to explore the functional involvement of individual or combined treatments on cell survival, we cultured 10 primary T-ALL samples in technical duplicate for 48 hours in complete medium in the presence of $\mathrm{IL}-7(50 \mathrm{ng} / \mathrm{mL}$, Peprotech), Methylprednisolon (50 ug/mL, Urbason, Sanofi), BEZ-235 (a dual PI3K/mTOR inhibitor, $800 \mathrm{nM}$ ) or without any treatment used as basal control. Cells were then stained with Annexin V/7-Aminoactinomycin D and absolute viable (and non-apoptotic) cell counts were performed by adding CountBright Absolute Counting Beads (Invitrogen) and calculated upon bead-based correction factor according to manufacturer's instructions.

\section{Mass cytometry}

Sample preparation

An average of $0.5-1 \times 10^{6}$ cells per condition were starved in X-VIVO medium $\left(37^{\circ} \mathrm{C}, 1\right.$ hour [h]), stimulated with IL-7 (50 ng/mL, 15 minutes [min]) or inhibited with BEZ-235 (800 nM, $30 \mathrm{~min}$ ) at $37^{\circ} \mathrm{C}$. Samples were fixed (MaxPar FIXI) and stored (10\% glycerol in fetal bovine serum, $-20^{\circ} \mathrm{C}$, adapted from Watson et $a l^{40}$ ).

\section{Sample staining}

Monoclonal antibody (moAb) characteristics are listed in the Online Supplementary Table S1. In-house antibody conjugations with palladium, indium and platinum isotopes were performed as described previously. ${ }^{41,42}$ Two sets of antibody cocktails (surface moAb, intracellular moAb) were prepared and handled as described previously. ${ }^{43}$ The samples were thawed, washed with MaxPar Cell Staining Buffer (CSB, 800 g, $5 \mathrm{~min}$, room temperature $[\mathrm{RT}]$ ), and barcoding moAb were added (15 min, RT). The cells were washed, pooled together and washed in CSB (800 g, $5 \mathrm{~min}, \mathrm{RT}$ ). In order to minimize technical variability between stimulated and unstimulated samples, differently treated samples from particular donors were barcoded, mixed and further processed in one tube. In order to control for the batch effect, each patient sample was acquired together with barcoded healthy donor PBMNC as an internal spike-in control. The samples were stained according to the MaxPar Phosphoprotein Staining with Fresh Fix (Fluidigm) protocol as recommended by the manufacturer.

\section{Sample acquisition}

The instrument (Helios, Fluidigm, CyTOF 6.7.1014 software) was prepared for acquisition according to the manufacturer's recommendation.

\section{Analysis}

Signal spillover between channels was corrected using the CATALYST $\mathrm{R}$ package as described previously. ${ }^{44} \mathrm{Com}-$ pensated fcs files were further processed using FlowJo (v10.5, FlowJo LLC) or Infinicyt v2.0 (Cytognos) software (Online Supplementary Figure S1). Heatmaps were created in MeV software ${ }^{45}$ by displaying arcsinh-transformed absolute values of median intensities. The fold change was calculated by subtracting the arcsinh-transformed median of unstimulated samples from the arcsinh-transformed median of stimulated samples.

\section{Vaevictis dimensionality reduction}

For visual projection of our data, we used the deep learning-based tool Vaevictis (https://github.com/ stuchly/vaevictis/), which combines ideas adapted from Szubert et $a .^{46}$ and Ding et al. ${ }^{47}$ with biased sampling. Using this approach, Vaevictis can produce reusable parametric mapping into the lower dimensional space to visualize both local and global relationships in very large datasets. Vaevictis dimensional reduction was calculated from a set of 13 parameters ( $p$-4E-BP1, p-STAT5, $p-A K T$, p-p38, p-S6, p-LCK, p-CREB-1, p-ERK1/2, p-RB, Ki-67, PTEN, MYC, and BCL-2).

\section{Molecular characterization of T-cell acute lymphoblastic leukemia samples}

The somatic mutation analysis was performed on primary genomic T-ALL DNA BM samples. PTEN mutation analysis was performed by polymerase chain reaction (PCR) sequencing of PTEN exon 7 and PCR products were directly sequenced in both directions using Applied Biosystems ABI PRISM-3130 Genetic Analyzer instrument (Life Technologies). Alignment was performed using the Basic Local Alignment Search Tool database (BLAST, www.blast.ncbi.nlm.nih.gov). IL-7R mutations/deletions were studied by Sanger sequencing of the PCR products of ILTR exon 6 (FW: TGCATGGCTACTGAATGCTC, RV: CCCACACAATCACCCTCTTT). NOTCH1 mutation analysis was performed by PCR amplification and direct DNA sequencing of exons 26, 27, 28 and part of exon 34 on TAD and PEST domains. ${ }^{48}$ The sequences were BLAST aligned and manually checked for mutations identification.

\section{Statistical analysis}

Mann-Whitney $U$ tests and Pearson correlation were performed in GraphPad Prism 5. A P-value below 0.05 was considered statistically significant.

\section{Results}

\section{T-cell acute lymphoblastic leukemia cells show constitutive activation of several signaling pathways relevant for proliferation, survival and translation activity}

We first evaluated the basal signaling profiles of proliferation and intracellular regulators in primary cells obtained 
Table 1. Clinical and biological characteristics of studied T-cell acute lymphoblastic leukemia patients

\begin{tabular}{|c|c|c|c|c|c|c|c|c|c|c|c|}
\hline Code & SEX & $\begin{array}{c}\text { AGE } \\
\text { (years) }\end{array}$ & $\begin{array}{c}\text { WBC } / \mathrm{mm}^{3} \\
\text { at diagnosis }\end{array}$ & $\begin{array}{l}\text { EGIL } \\
\text { score }\end{array}$ & $\begin{array}{c}\text { ETP immuno- } \\
\text { phenotype }\end{array}$ & $\begin{array}{c}\text { PDN } \\
\text { response }\end{array}$ & $\begin{array}{c}\text { MRD } \\
\text { at day 15* }\end{array}$ & PTEN & $\begin{array}{l}\text { NUP214 } \\
\text { /ABL1 }\end{array}$ & $\begin{array}{c}\text { IL7R } \\
\text { exon } 6\end{array}$ & $\begin{array}{l}\text { NOTCH1 (exons } \\
\text { 26-27-28-34T- } \\
\text { P)** }\end{array}$ \\
\hline TALL-1 & $M$ & 14 & 88,900 & T-IV & no & PGR & nd & wt & wt & nd & nd \\
\hline TALL-2 & $M$ & 14 & 169,570 & TI/II & no & PPR & $30.0 \%$ & mut & wt & wt & wt \\
\hline TALL-3 & $F$ & 9 & 136,720 & T-III & no & PGR & nd & mut & wt & wt & mut \\
\hline TALL-4 & $M$ & 6 & 60,050 & T-III & no & PGR & nd & wt & wt & wt & mut \\
\hline TALL-5 & $\mathrm{F}$ & 12 & 400,000 & T-III & no & PGR & $2.0 \%$ & mut & wt & wt & wt \\
\hline TALL- 6 & $\mathrm{~F}$ & 13 & 1026,000 & T-IV & no & PPR & $70.0 \%$ & mut & wt & wt & wt \\
\hline TALL-8 & $\mathrm{F}$ & 5 & 362,600 & T-III & no & PGR & $1.15 \%$ & wt & wt & wt & mut \\
\hline TALL-9 & M & 6 & 14,420 & T-III & no & PGR & $0.01 \%$ & wt & wt & wt & mut \\
\hline TALL-10 & $M$ & 14 & 168,090 & T-IV & no & PPR & $24.0 \%$ & mut & wt & wt & wt \\
\hline TALL-11 & $M$ & 17 & 15,800 & T-III & no & PGR & $0.14 \%$ & wt & wt & wt & mut \\
\hline TALL-12 & $M$ & 11 & 159,580 & TI/II & no & PPR & $23.0 \%$ & wt & mut & wt & wt \\
\hline TALL-13 & $M$ & 8 & 14,630 & T-III & no & PGR & $0.14 \%$ & wt & wt & wt & mut \\
\hline TALL-14 & $M$ & 3 & 369,810 & T-III & no & PPR & $61.0 \%$ & mut & wt & wt & wt \\
\hline TALL-15 & $M$ & 11 & 9,390 & TI/II & no & PGR & $1.30 \%$ & wt & mut & wt & mut \\
\hline TALL-16 & $\mathrm{F}$ & 17 & 1,700 & $\mathrm{TI} / \mathrm{II}$ & yes & PPR & $90.0 \%$ & wt & wt & wt & wt \\
\hline TALL-17 & $M$ & 8 & 176,000 & T-III & no & PPR & $0.99 \%$ & wt & wt & wt & wt \\
\hline
\end{tabular}

TALL: T-cell acute lymphoblastic leukemia; PDN: prednisone; PGR: prednisone good responder; PPR: prednisone poor responder; IR: intermediate risk; HR: high risk; SR: standard risk; wt: wild-type; mut: mutated; ETP: early T-cell phenotype according to Coustan-Smith et al. ${ }^{61}$ *According to the criteria by Basso et al. ${ }^{62}$ the $\%$ of flow cytometry minimal residual disease (MRD) in the bone marrow of day 15 identifies 3 patient's risk groups: standard risk (SR, 0.1\% blasts), intermediate risk (IR, 0.1 to 10\%), and high risk (HR, $\geq 10)$; nd: not determined. $* \star$ Details on the specific exons associated to mutation are reported in the Online Supplementary Table S3.

from the BM or PB of 16 pediatric T-ALL diagnostic specimens, 14 of which were reported previously. ${ }^{38}$ The clinical and laboratory characteristics of the patients are described in Table 1. Using cytometry by time of flight (СуTOF), we analyzed the samples with a panel of 30 moAb, including eight CD45 barcodes, 15 surface markers and 15 intracellular regulators of various pathways and cellular processes, such as the PI3K-AKT-mTOR, JAK-STAT, MEK-ERK, MAPK pathways, and the activation of T-cell receptors, the cell cycle, transcription and apoptosis (Table 2; Online Supplementary Table S1). Viable T-ALL cells were gated separately from residual non-malignant T cells (Online Supplementary Figure S1). T cells obtained from healthy donor PB $(n=3)$ were used as controls.

Compared to their healthy T-cell counterparts, T-ALL cells showed constitutive activation of several functional markers: phosphorylated forms of 4E-BP1, AKT and S6 (PI3K-AKT-mTOR pathway members); Ki-67, a cell cycle progression marker; $p-R B$, a regulator of the $\mathrm{G} 1$ to $S$ transition; $p$-ERK1/2 and p-p38, members of the mitogen-activated kinase (MAPK) family; and CREB, a transcription factor regulating proliferation, differentiation and survival (Figure 1).

In contrast, the expression of BCL-2 (regulating anti-apoptotic activity) was significantly lower in T-ALL cells. Overall the phospho-signature and levels of intracellular regulators was similar in healthy donors' peripheral T cells and residual nonmalignant $T$ cells (internal control) and distinct from T-ALL.

\section{Single-cell analysis of functional parameters reveals intra- and interindividual heterogeneity of T-cell acute lymphoblastic leukemia cells}

In order to visualize the internal heterogeneity of the signaling network in T-ALL cells at the phospho-proteomic level, we applied deep learning-based Vaevictis dimensionality reduction (Figure $2 \mathrm{~A}$ ). Signaling, proliferation and internal regulators (13 parameters) were considered for the projection calculation. In the presented cohort of pediatric T-ALL samples, we observed heterogeneity in the signaling regulators, however there was a dominant subset (>50\%) within the T-ALL cell compartment in the 
Table 2. Cytometry by time of flight (CyTOF) panel

\begin{tabular}{|c|c|c|c|c|c|c|}
\hline Target & Metal & Clone & Function & $\begin{array}{l}\text { Detection prior or after } \\
\text { metOH permeabilization }\end{array}$ & Vendor & Catalogue number \\
\hline CD45 & $89 Y$ & $\mathrm{HI} 30$ & barcoding & prior & Fluidigm & $3089003 B$ \\
\hline CD45 & $104 \mathrm{Pd}$ & $\mathrm{Hi} 30$ & barcoding & prior & H.Mei & NA \\
\hline CD45 & $106 \mathrm{Pd}$ & $\mathrm{Hi} 30$ & barcoding & prior & H.Mei & NA \\
\hline CD45 & $108 \mathrm{Pd}$ & $\mathrm{Hi} 30$ & barcoding & prior & H.Mei & NA \\
\hline CD45 & $110 \mathrm{Pd}$ & Hi30 & barcoding & prior & H.Mei & NA \\
\hline HLA-I & 113 In & W6/32 & immunophenotype & prior & Exbio & $11-422-\mathrm{C} 100$ \\
\hline CD45 & $115 \mathrm{In}$ & MEM-28 & barcoding & prior & Exbio & $11-222-\mathrm{M} 001$ \\
\hline CD19 & $142 \mathrm{Nd}$ & HIB19 & immunophenotype & prior & Fluidigm & 3142001B \\
\hline CD5 & $143 \mathrm{Nd}$ & UCHT2 & immunophenotype & prior & Fluidigm & $3143007 \mathrm{~B}$ \\
\hline CD4 & $145 \mathrm{Nd}$ & MEM-241 & immunophenotype & prior & Exbio & $11-359-C 100$ \\
\hline CD8a & $146 \mathrm{Nd}$ & RPA-T8 & immunophenotype & prior & Fluidigm & 3146001B \\
\hline CD7 & $147 \mathrm{Sm}$ & CD7-6B7 & immunophenotype & prior & Fluidigm & 3147006B \\
\hline CD34 & $148 \mathrm{Nd}$ & 581 & immunophenotype & prior & Fluidigm & 3148001B \\
\hline p-4E-BP1 [T37/T46] & $149 \mathrm{Sm}$ & 236B4 & phospho-protein & after & Fluidigm & $3149005 A$ \\
\hline p-Stat5 [Y694] & $150 \mathrm{Nd}$ & 47 & phospho-protein & after & Fluidigm & $3150005 \mathrm{~A}$ \\
\hline CD2 & 151Eu & $\mathrm{TS} 1 / 8$ & immunophenotype & prior & Fluidigm & 3151003B \\
\hline p-Akt [S473] & $152 \mathrm{Sm}$ & D9E & phospho-protein & after & Fluidigm & $3152005 A$ \\
\hline BCL-2 & 153Eu & $\mathrm{Bcl}-2 / 100$ & apoptosis & after & Exbio & $11-668-C 100$ \\
\hline CD56 & $155 \mathrm{Gd}$ & B159 & immunophenotype & prior & Fluidigm & $3155008 B$ \\
\hline p-p38 [T180/Y182] & $156 \mathrm{Gd}$ & D3F9 & phospho-protein & after & Fluidigm & $3156002 A$ \\
\hline TSLP-R & $158 \mathrm{Gd}$ & 1B4 & immunophenotype & prior & Fluidigm & $3158026 \mathrm{~B}$ \\
\hline$p-S 6$ [S240/244] & 159Tb & D68F8 & phospho-protein & after & CST & 5364 \\
\hline CD38 & $160 \mathrm{Gd}$ & HIT2 & immunophenotype & prior & Exbio & $11-366-C 100$ \\
\hline CD3 & 161Dy & UCHT1 & immunophenotype & prior & Exbio & $11-514-C 100$ \\
\hline$p-L c k$ [T505] & 162Dy & 4/LCK-Y505 & phospho-protein & after & Fluidigm & $3162004 \mathrm{~A}$ \\
\hline Tdt & 164Dy & E17-1519 & immunophenotype & after & Fluidigm & $3164015 B$ \\
\hline$p-C R E B$ [S133] & $165 \mathrm{Ho}$ & $87 G 3$ & phospho-protein & after & Fluidigm & $3165009 A$ \\
\hline $\mathrm{p}-\mathrm{Rb}$ [S807/811] & $166 \mathrm{Er}$ & $\mathrm{J} 112-906$ & proliferation & after & Fluidigm & $3166011 \mathrm{~A}$ \\
\hline $\mathrm{Ki}-67$ & $168 \mathrm{Er}$ & B56 & proliferation & after & Fluidigm & $3168007 \mathrm{~B}$ \\
\hline CD33 & 169Tm & WM53 & immunophenotype & prior & Fluidigm & $3169010 B$ \\
\hline Cl.Caspase3 (D175) & $170 \mathrm{Er}$ & poly & apoptosis & after & CST & 9661 \\
\hline p-Erk1/2 [T202/Y204] & $171 Y b$ & $\mathrm{D} 13.14 .4 \mathrm{E}$ & phospho-protein & after & Fluidigm & $3171010 \mathrm{~A}$ \\
\hline PTEN & $172 \mathrm{Yb}$ & A2B1 & tumor supressor & after & $\mathrm{BD}$ & 559600 \\
\hline HLA-DR & $174 \mathrm{Yb}$ & L243 & immunophenotype & prior & Fluidigm & 3174001B \\
\hline c-Myc & $176 \mathrm{Yb}$ & 9E10 & transcription factor & after & Fluidigm & $3176012 B$ \\
\hline DNA & $191|r / 193| r$ & & DNA intercalator & after & Fluidigm & $201192 B / 201192 A$ \\
\hline CD45 & $194 \mathrm{Pt}$ & MEM-28 & barcoding & prior & Exbio & $11-222-\mathrm{M} 001$ \\
\hline HLA-I & $198 \mathrm{Pt}$ & $w 6 / 32$ & barcoding & prior & Bxcell & BE0079 \\
\hline
\end{tabular}

CST: cell signaling technology; BD: BD Biosciences; metOH: methanol. 

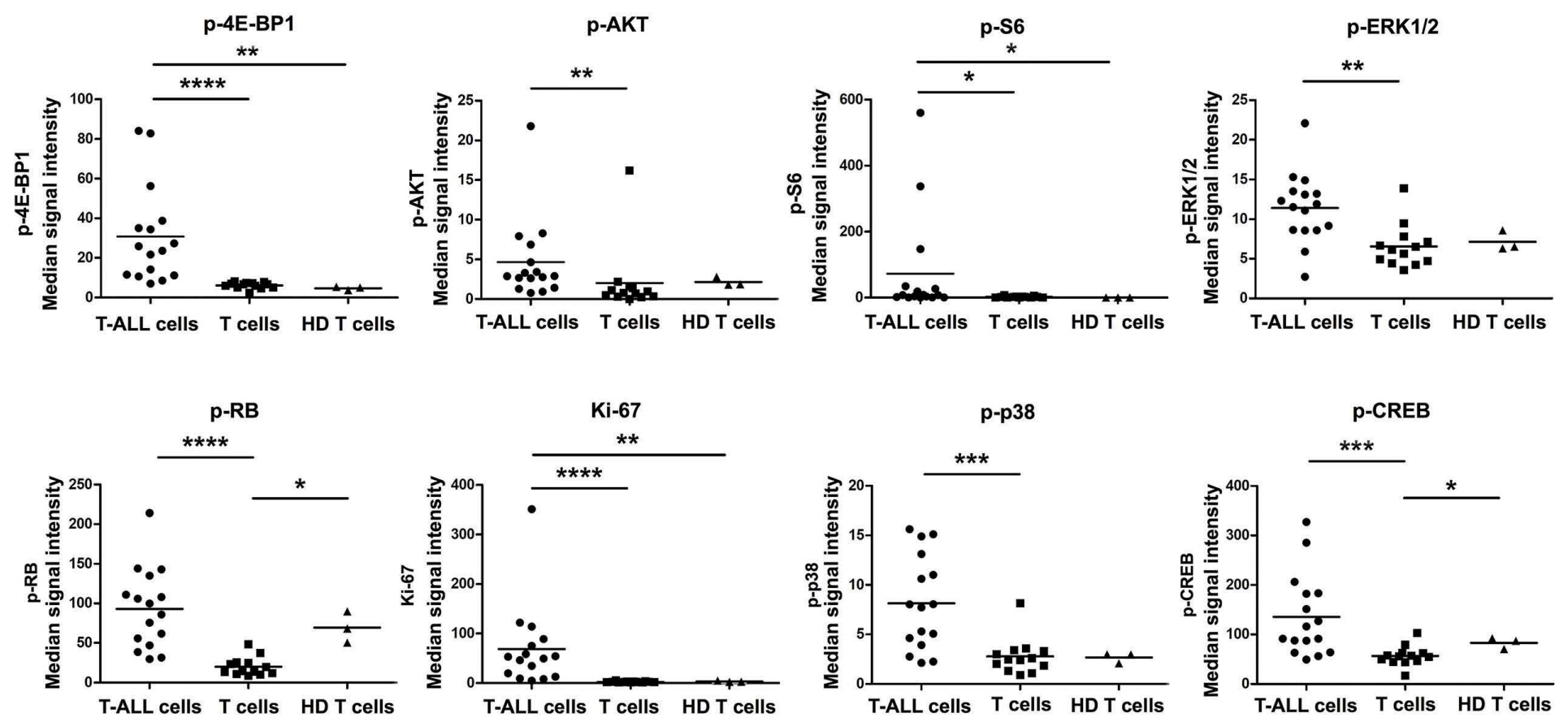

PTEN

p-STAT5

p-LCK
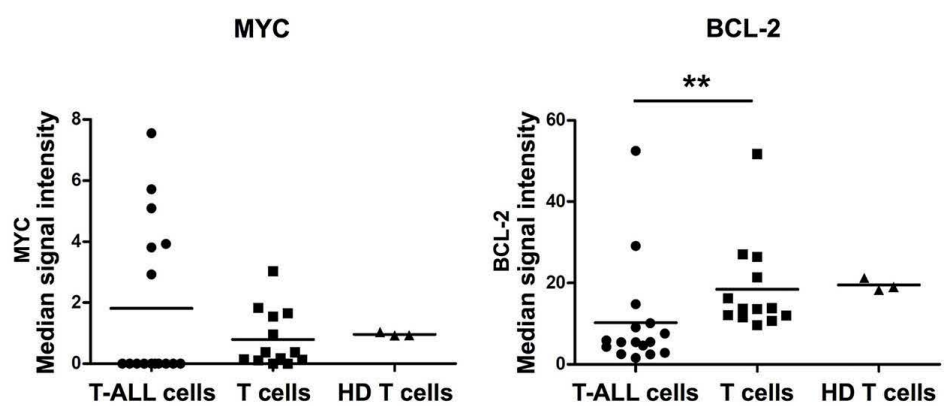

Figure 1. Basal signaling profile of T-cell acute lymphoblastic leukemia and non-malignant $\mathbf{T}$ cells. T-cell acute lymphoblastic leukemia (T-ALL) cells show constitutive activation of various signaling pathways as well as proliferation markers as compared to either residual non-malignant $T$ cells or T cells isolated from healthy donor's (HD) peripheral blood (PB). Median intensity evaluated by mass cytometry of p-4E-BP, p-AKT, p-S6, p-ERK1/2 (top panels); p-RB, Ki-67, p-p38, p-CREB (middle panels); PTEN, p-STAT5, p-LCK, MYC and BCL-2 (bottom panels) in gated T-ALL cells obtained from bone marrow (BM) or PB of T-ALL patients $(n=16)$, residual T cells from T-ALL patients $(n=13)$ and T cells obtained from HD PB $(n=3)$ are shown. T-ALL cells, residual T cells and HD T cells are depicted as circles, squares and triangles, respectively. ${ }^{\star} P<0.05, \star \star * P<0.01, * \star \star P<0.001, * \star \star \star x<0.0001$.

majority of T-ALL patient specimens (Figure 2A; Online Supplementary Figure S2). In addition to the dominant subset, minor subsets of T-ALL cells were identified with a continuous transition between individual subsets. Notably, in some patients (e.g., TALL-10), a subset of T-ALL cells is clearly separated from the major subset, suggesting an intrinsic heterogeneity of T-ALL cells.

We then compared interindividual differences in T-ALL patients' signaling, proliferation and intracellular regulators. We observed a similar pattern in six PTEN exon 7 mutated (PTENmut) T-ALL patients (Figure 2A). Interestingly, on overlaid Vaevictis projections (Figure 2B), PTENmut patients are located in the same compartment of the map, forming a cluster. T-ALL samples with NUP214/ABL1 gene fusion are located within the same compartment but do not separate from PTEN wild-type (PTENwt) cells. As expected, PTENmut cells showed the absence of PTEN protein and a significantly higher MYC expression than PTENwt cells (Figure 2C). PTENwt cells showed only partial expression of MYC, documenting the maintenance of PTEN-MYC axis regulation. We also observed decreased levels of Ki-67 and p-p38 MAP kinase in PTENmut T-ALL patients. Finally, T-ALL patients with the NUP214/ABL1 gene fusion showed constitutive activation of the JAKSTAT pathway, documented as P-STAT5, compared to TALL patients without this gene fusion (Figure 2C). The impact of both PTEN and MYC expression on the pattern of signaling, proliferation and intracellular regulators is shown on the Vaevictis map (Online Supplementary Figures S3 and S4).

Further, we analyzed the correlation of MYC expression with NOTCH1 status in 15 of the 16 studied patients. The distribution of MYC expression among NOTCH1wt cases was variable with two clear clusters, one low (3 cases) and one high (5 cases) as illustrated in Figure 2D. Of the seven NOTCH1mut cases, six were MYC low and one was very high. We then analyzed the correlation between NOTCH1 and PTEN status. Six cases were PTENwt/NOTCH1mut, one case was PTENmut/NOTCH1mut, five cases PTENmut/NOTCH1wt and three cases PTENwt/NOTCH1wt (Table 1). As shown in Figure 2D, the level of MYC was low in all PTENwt cases regardless of NOTCH1 status ( 3 cases NOTCH1wt and 6 cases NOTCH1mut), whereas all PTENmut cases displayed high 
MYC (5 cases NOTCH1wt and 1 case NOTCH1mut). In order to further investigate the intrinsic heterogeneity observed in the Vaevictis projections, we analyzed the TALL-10 sample with the most pronounced separated subsets, and we identified mosaic expression of both PTEN and MYC proteins. On the contrary homogeneous expression of these two proteins was observed in representative PTENwt (TALL-11) or PTENmut (TALL-14) patients (Figure $2 \mathrm{E}$ and $\mathrm{F}$ ), indicating that $\mathrm{PTEN}-\mathrm{MYC}$ regulation can be maintained even at a subclonal level in T-ALL. On the other hand, no differences in the phosphorylation of p38 or the expression of Ki-67 were observed in the two subclones of TALL-10 patient.

Finally, in order to confirm the reliability of the approach, we evaluated interindividual variability in the signaling profiles of both proliferation and intracellular regulators in residual $T$ cells (Online Supplementary Figure S4) and compared it to that of T-ALL cells. Residual T cells showed very low interpatient variability, while T-ALL cells showed a high degree of intersample heterogeneity, indicating that the activation signatures in T-ALL blasts were disease-specific and not caused by sample handling or processing.
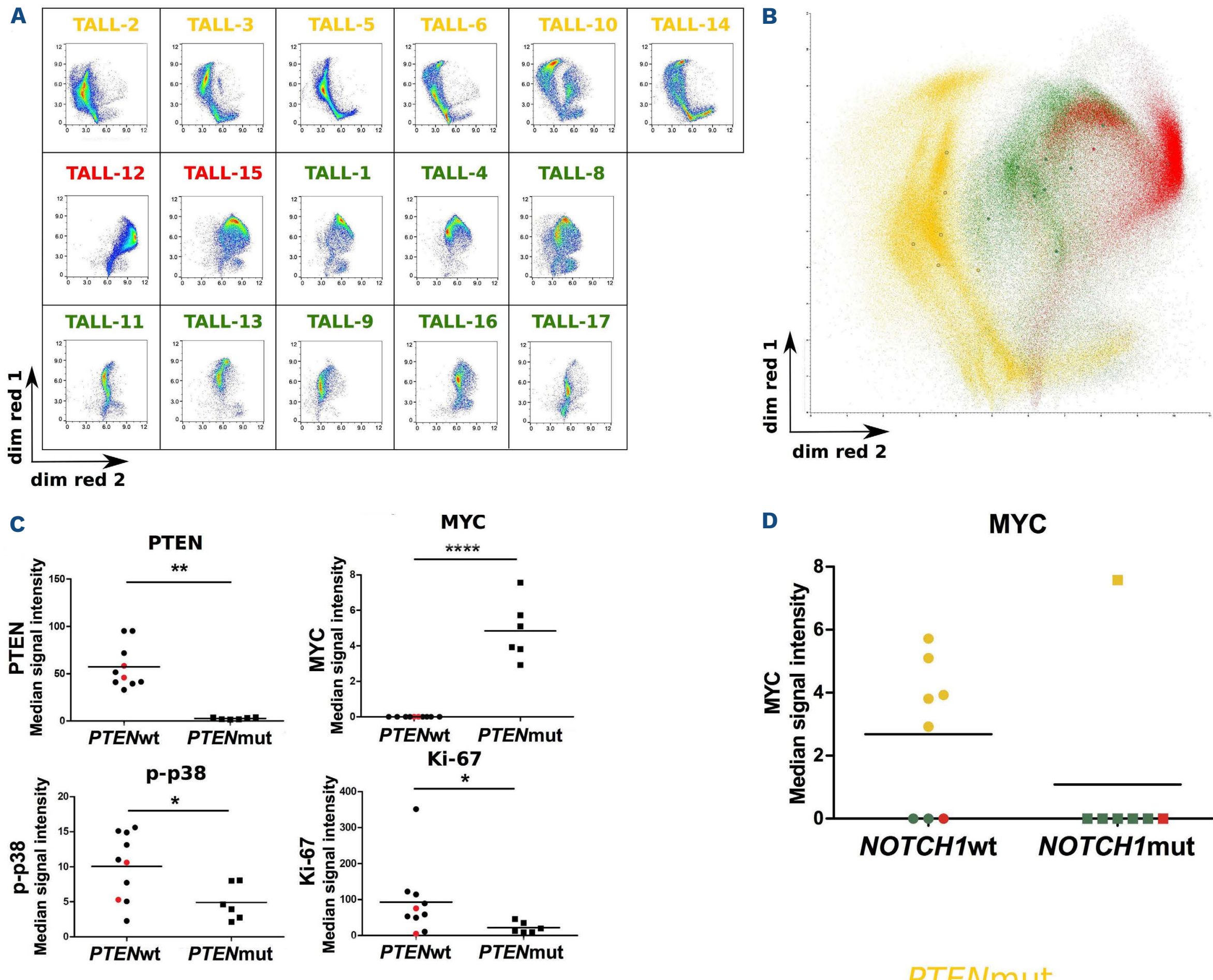

D

MYC
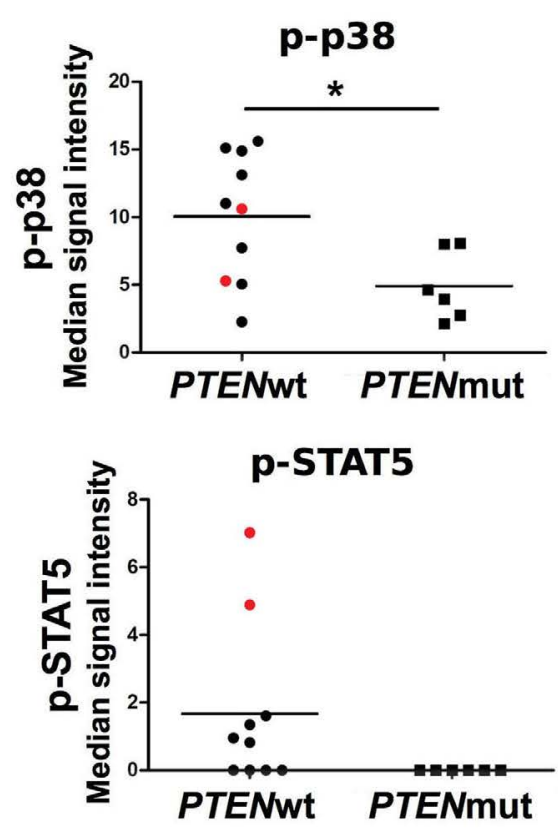

\footnotetext{
- NUP214/ABL1 pos T-ALL cells

- NUP214/ABL1 neg T-ALL cells

- PTENmut T-ALL cells
}
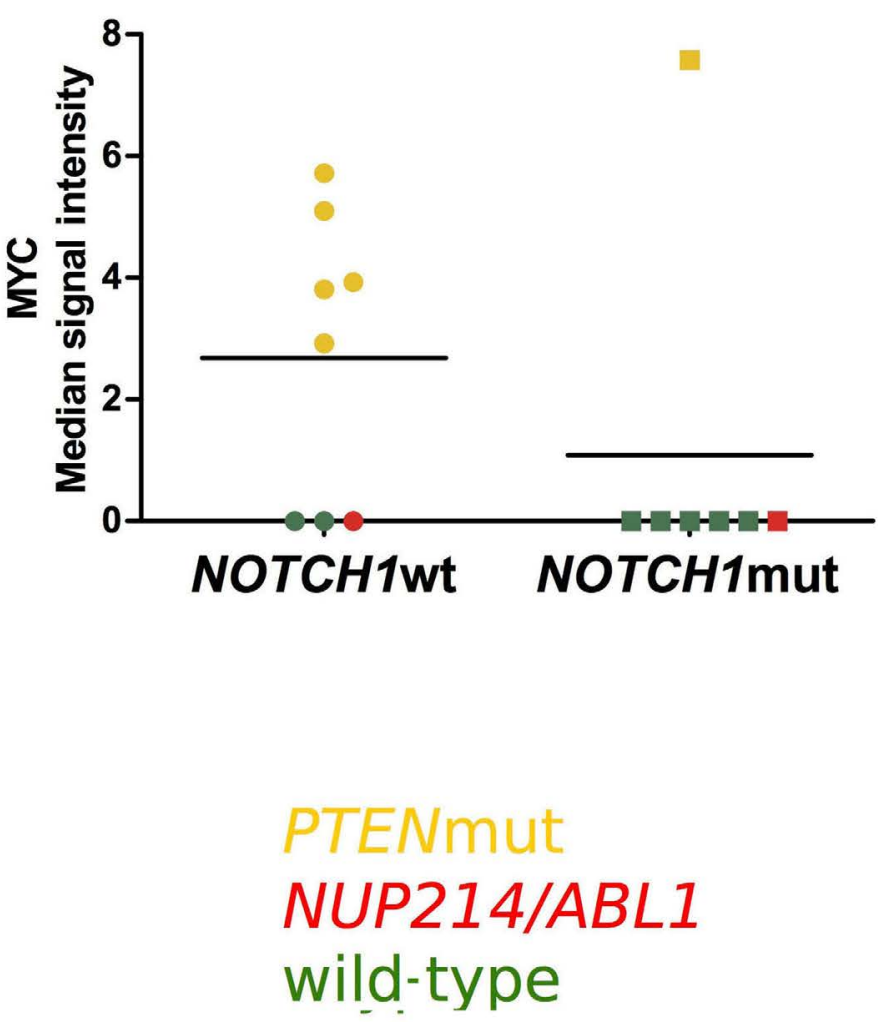

Figure 2. Continued on following page. 
E
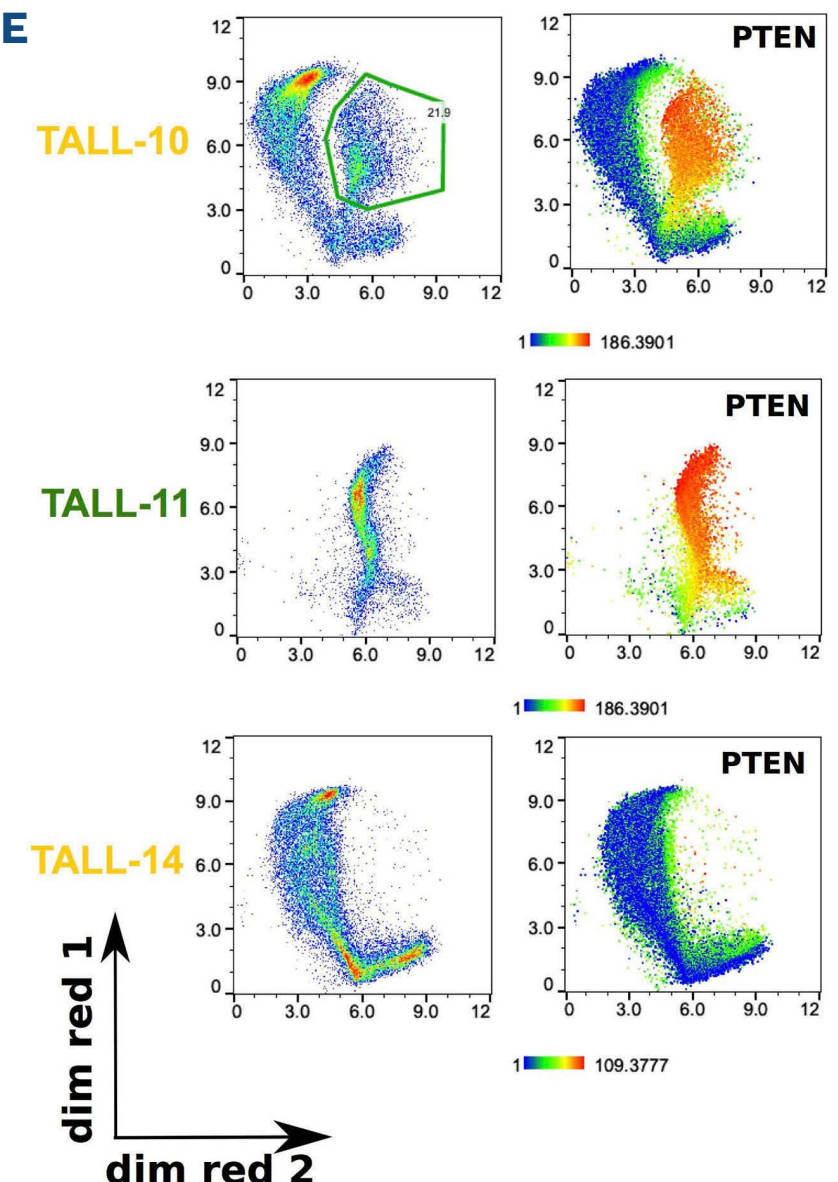

$1=186.3901$

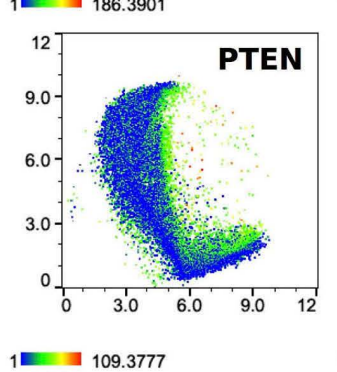

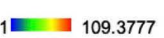

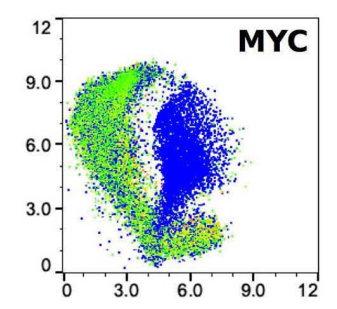

$1=42.0802$

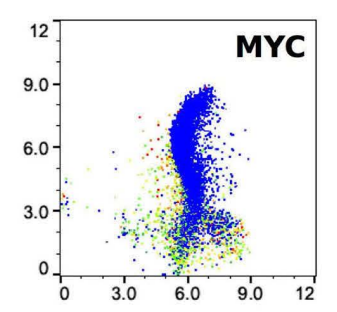

$1=12.0802$

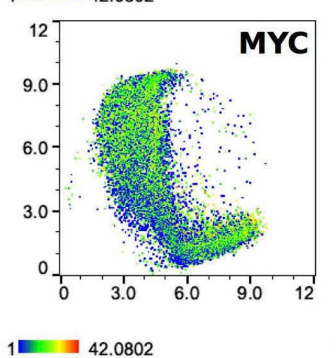

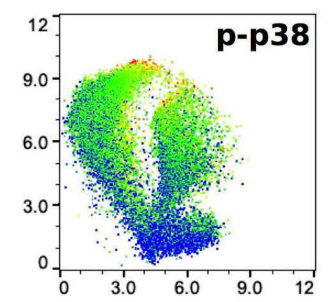

$1=253.2837$

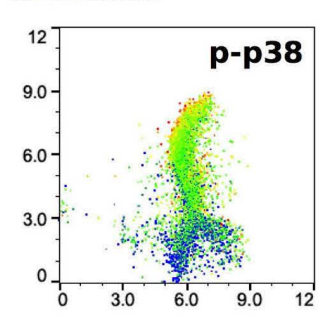

$1=253.2837$

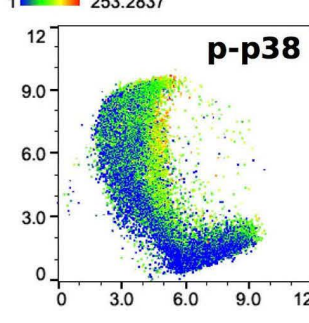

$1=237.3173$

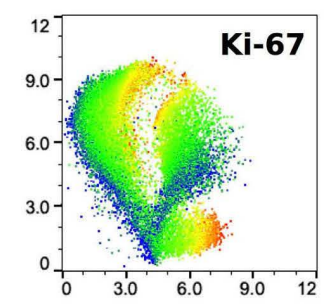

$1=1555.0446$

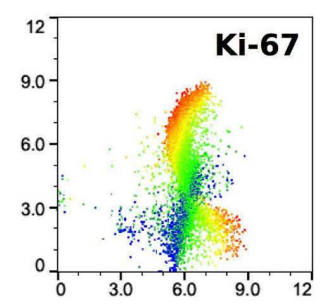

$1=1555.0446$

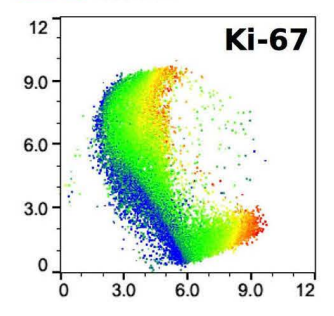

$\mathbf{F}$
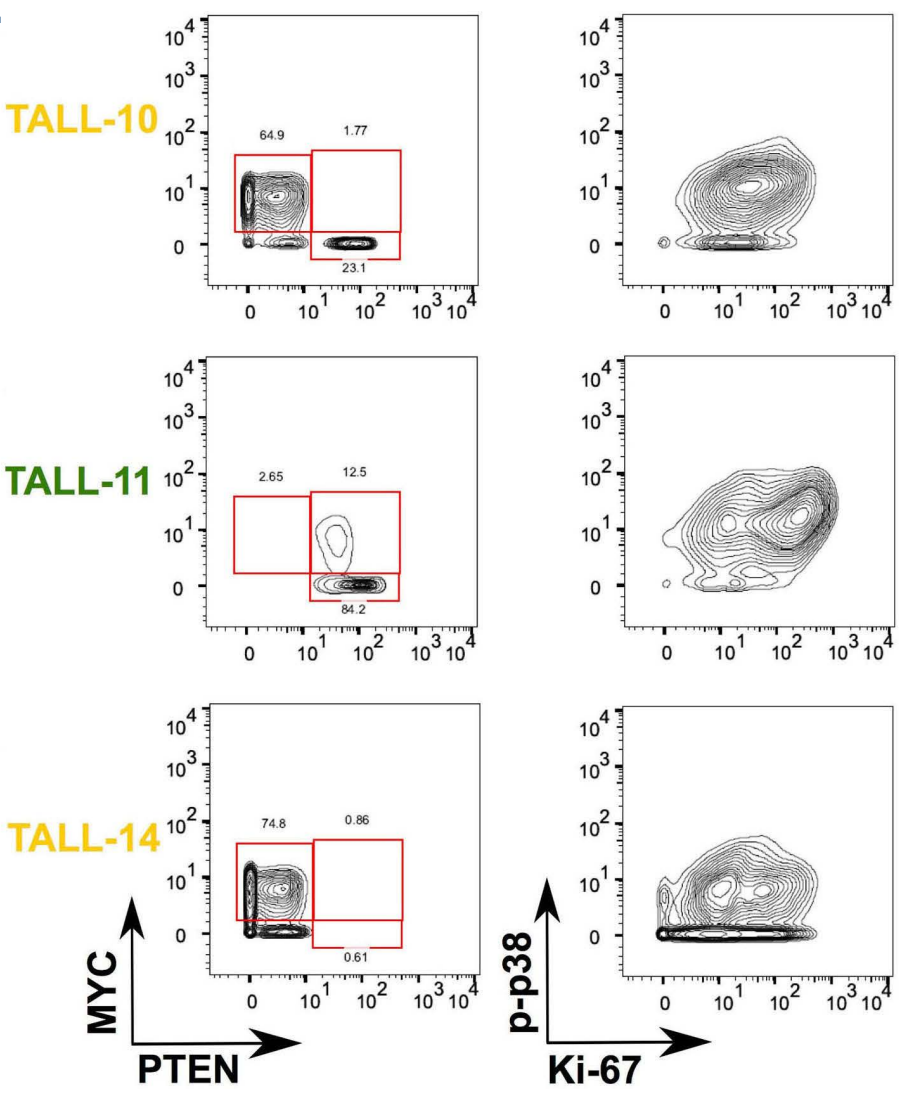

Figure 2. Single-cell and bulk analysis of functional parameters reveals intra- and interindividual heterogeneity of T-cell acute lymphoblastic leukemia cells.T-cell acute lymphoblastic leukemia (T-ALL) cells from pediatric patients with different genetic features are depicted: PTEN exon 7 mutated (PTENmut) T-ALL ( $\mathrm{n}=6$, orange label), T-ALL harboring NUP214/ABL1 gene fusion ( $\mathrm{n}=2$, red label) and T-ALL wild-type (wt) for both PTEN mutation and NUP214/ABL1 gene fusion ( $\mathrm{n}=8$, green label). (A) Deep learningbased Vaevictis dimensionality reduction (dim red) was applied on T-ALL cells obtained from 16 pediatric T-ALL patients at diagnosis. Signaling, proliferation and internal regulators (PTEN, MYC, BCL-2, p-4E-BP1, p-STAT5, p-AKT, p-p38, p-S6, p-LCK, p-CREB, p-RB, Ki-67, p-ERK1/2) were considered for the projection calculation. (B) Overlaid Vaevictis projection based on signaling, proliferation and internal regulators of T-ALL cells obtained from T-ALL patients $(n=16)$ at diagnosis. Circles represent medians of particular T-ALL specimens. (C) Median intensity of deregulated proteins in PTENmut T-ALL samples ( $\mathrm{n}=6$, squares) as compared to PTENwt samples ( $n=10$, circles) are shown. T-ALL samples carrying NUP214/ABL1 gene fusion $(n=2)$ are depicted as red circles. (D) Median intensity of MYC protein in NOTCH1wt (left) and NOTCH1 mutated (NOTCH1mut) (right) patients. (E) The expression of the most deregulated proteins in PTENmut samples are shown. In the Vaevictis projection of T-ALL phospho-signature a compact sub-clone in TALL-10 specimen was identified (green gate). For comparison, 1 representative PTENmut (TALL-14, yellow label) and one representative PTENwt (TALL-11, green label) T-ALL sample are shown. Heat of each map corresponds with expression of indicated marker. Note the scale max differ for each marker and each sample. Mosaic expression of both, PTEN and MYC proteins were observed, while no differences in phosphorylation of p38 and expression of Ki-67 are present in the two sub-clones of TALL-10 patient. (F) Contour plots showing expression of PTEN, MYC, p-p38 and Ki-67 in TALL-10 in comparison with TALL-11 and TALL-14 samples are shown. 
In vitro response to IL-7 correlates with higher proliferation and predicts good response to prednisone induction

The IL-7/IL-7R signaling axis activates three main pathways in T cells, the JAK-STAT, PI3K-AKT-mTOR and MEKERK pathways, ultimately promoting leukemia cell viability, cell cycle progression and growth. The contribution of each of these pathways to a particular functional outcome is still not completely known and appears to differ between normal and malignant states. ${ }^{20}$ For these reasons, we investigated the impact of IL-7/IL-7R signaling on the modulation of the before mentioned functional nodes upon IL-7 stimulation.

For this purpose, we analyzed IL-7-induced ex vivo perturbation of nine phospho-epitopes in T-ALL cells, residual $T$ cells and $T$ cells obtained from healthy donor's PB (Figure 3A). We resolved two response types: (i) IL-7-responsive T-ALL samples $(n=6)$ and (ii) IL-7-non-responsive T-ALL samples $(n=10)$, distinguished by their difference in p-STAT5 activation. P-STAT5 increased by 2.5 -fold in IL-7 responders, while it remained unchanged in IL-7 non-responders. The fold increase in P-STAT5 was caused solely by IL-7 induction (Figure 3B), since the basal levels were comparable in both groups (Online Supplementary Figure S5). In contrast, in normal T cells, we observed a homogeneous pattern of the p-STAT5 response in 14 of 15 samples (Figure 3A; Online Supplementary Figure S6). However, apart from p-STAT5, no other tested nodes were differentially expressed between the two subgroups (Online Supplementary Figure S7). We next investigated the correlation between IL-7-induced STAT5 phosphorylation and the expression of the IL-7R in T-ALL cells $(n=15)$ and residual T cells $(n=9)$. The fold change in P-STAT5 upon IL7 stimulation quantitatively correlated with the percentage of CD127 (Figure 3C) but not with CD132 (common $\gamma$ chain, a subunit of the IL-7 receptor, Online Supplementary Figure S8) expression. However, the IL-7 non-responsiveness of T-ALL blasts cannot be explained solely by the lower expression of CD127 or CD132. For example, cases TALL-15 and TALL-9 expressed CD127 at 60\% and >90\% respectively but responded poorly to IL-7. TALL-15 was harboring NUP214/ABL1 gene fusion with basal P-STAT5 already at the maximal level, which can explain its non-responsiveness. By contrast TALL-9 (PTENwt, IL-7Rwt and NUP214/ABL1wt) displayed low responsiveness despite its low basal P-STAT5. Yet, STAT-5 was phosphorylated after pervanadate treatment showing that the method worked and also that STAT- 5 could potentially be phosphorylated in these cells. Thus the lack of responsiveness to IL-7 in this specific case remains unclear.

In order to further investigate the characteristics of IL-7 non-responsive T-ALL samples, we compared the profiles

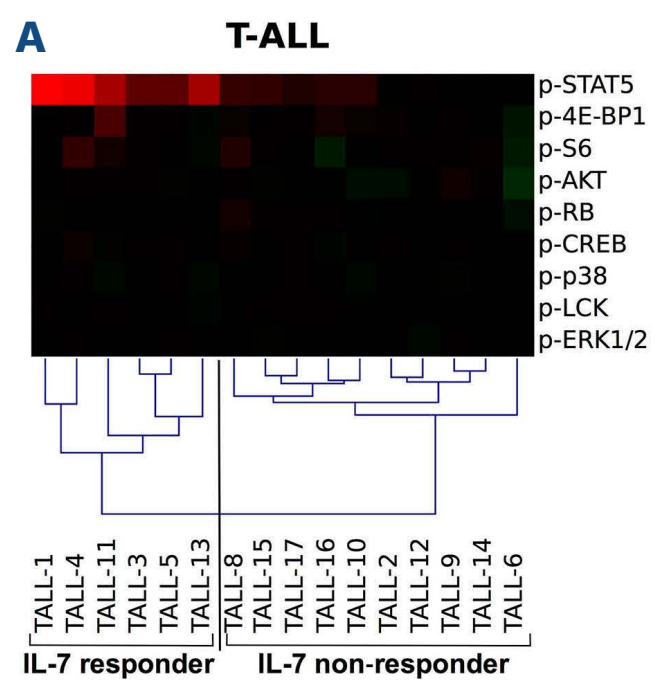

B

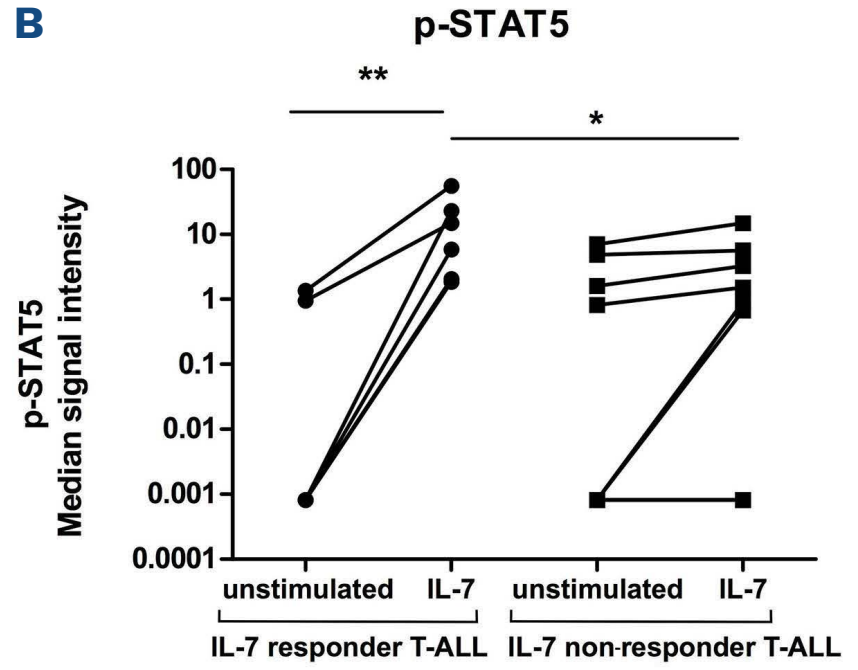

\section{T cells}
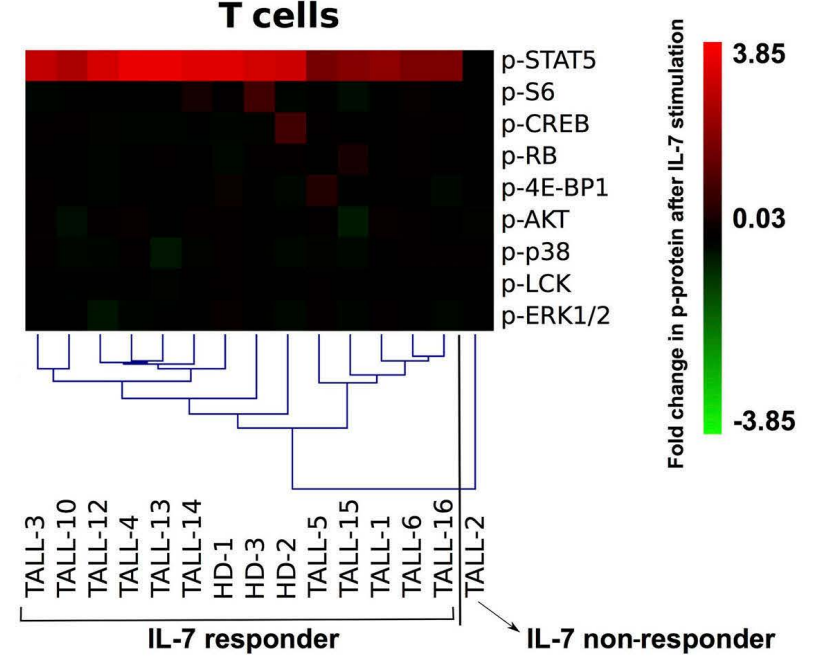

IL-7 responder IL-7 non-responder
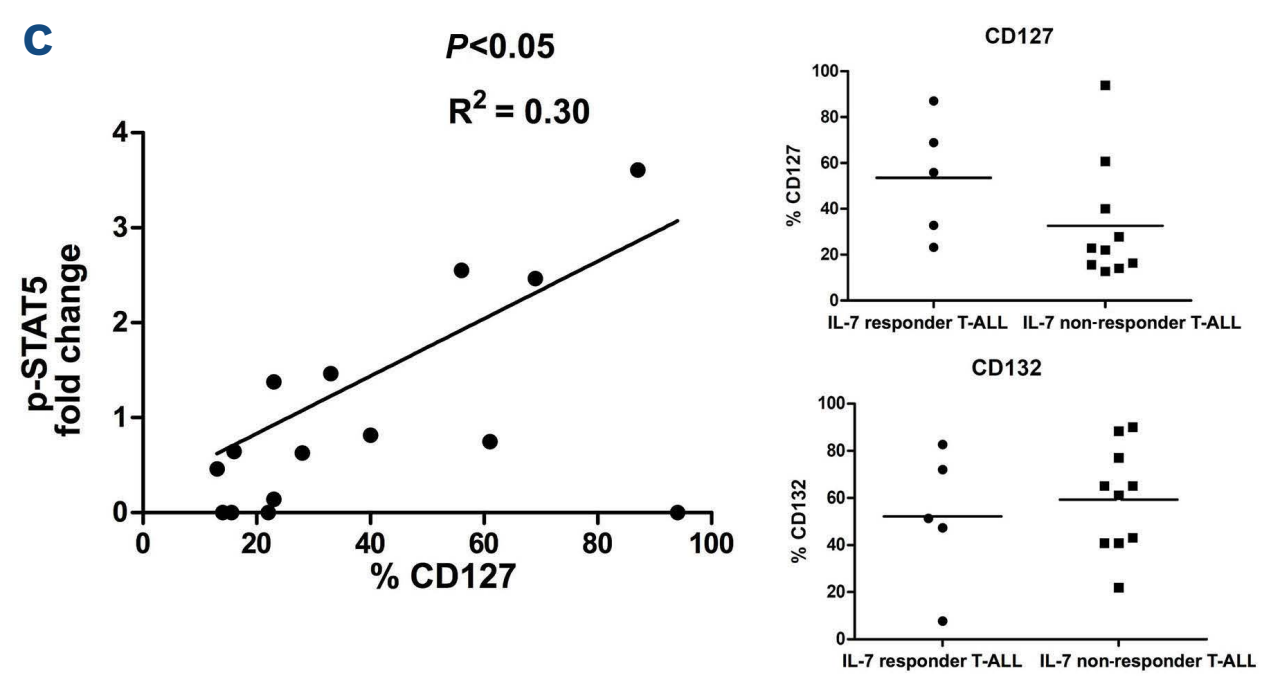

Figure 3. Continued on following page. 

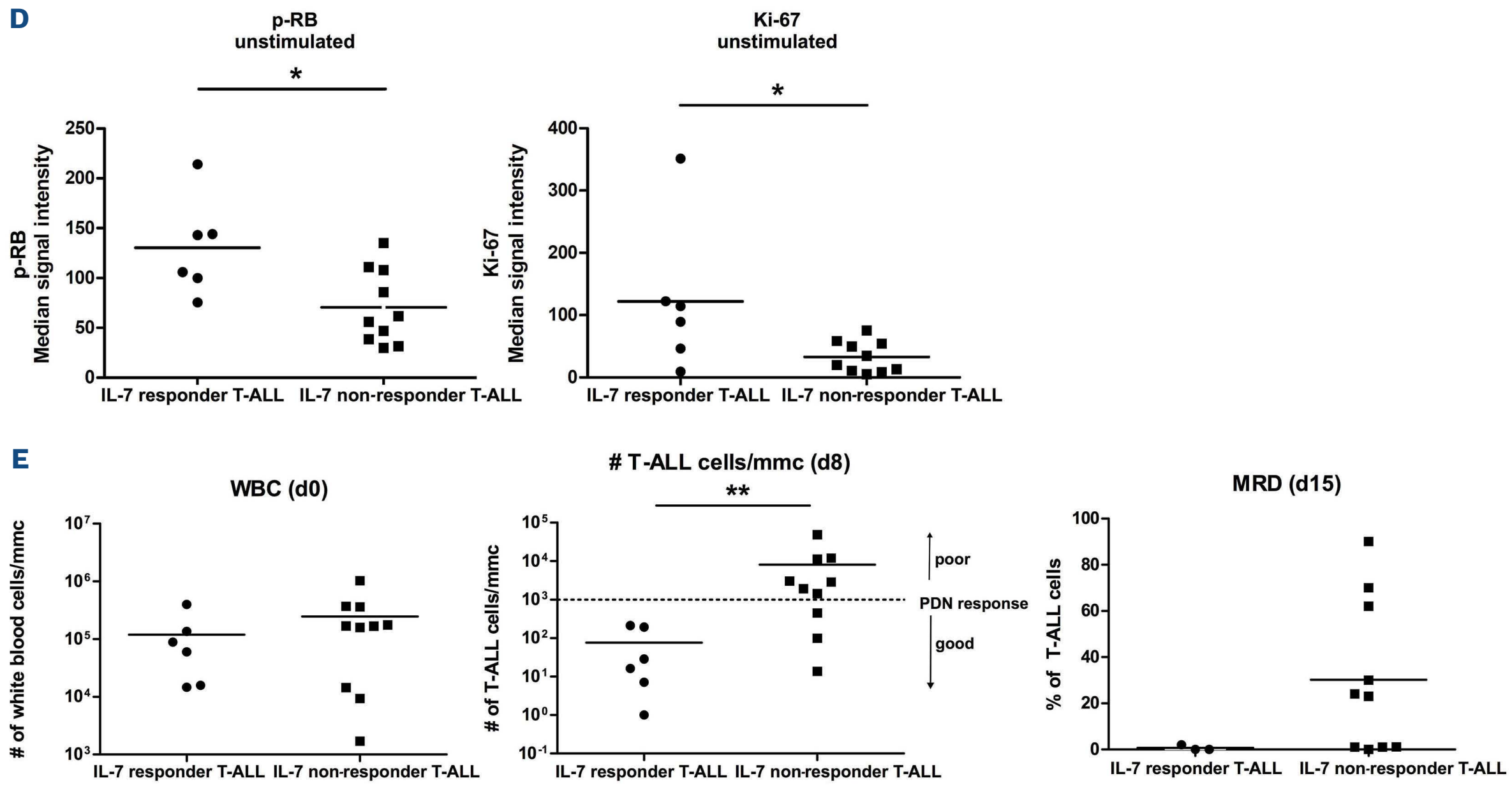

Figure 3. Response to IL-7 of T-cell acute lymphoblastic leukemia cells and non-malignant T cells. (A) Heatmap represents fold change in protein phosphorylation in ex vivo IL-7 stimulated cells as compared to their unstimulated state in T-cell acute lymphoblastic leukemia (T-ALL) cells (left) and residual T cells (right) obtained from T-ALL patients and healthy donors (in columns) at diagnosis. Heat in the squares represents the fold change in phosphorylation of proteins listed in rows. Hierarchical clustering with Euclidean distance metrics and an average linkage dissects two clusters of T-ALL and residual T cells: IL-7 responder and IL-7 non-responder. Values of 0 were replaced with 0.0001. (B) Median intensity of p-STAT5 in untreated and ex vivo IL-7 stimulated specimens from IL-7 responder T-ALL (circles, $n=6$ ) and IL-7 non-responder T-ALL (squares, 10) is shown. Median intensity of $p-$ STAT5 increased after ex vivo IL-7 stimulation in IL-7 responder T-ALL but not in IL-7 non-responder.(C) Correlation between percentage of surface CD127 (IL-7RA) expression evaluated by flow cytometry and fold change of p-STAT5 after ex vivo IL-7 stimulation in T-ALL samples obtained from diagnostic T-ALL specimens. No difference in percentage of CD127 or CD132 (common $\gamma$ chain, subunit of IL-7R) surface expression was found between IL-7 responder and IL-7 non-responder T-ALL. (D) Median intensity of $\mathrm{RB}$ phosphorylation (left) and Ki-67 expression (right) in unstimulated specimens from IL-7 responder T-ALL (circles, $\mathrm{n}=6$ ) and IL-7 non-responder T-ALL (squares, $n=10$ ) are shown. (E) White blood cell count (WBC) at diagnosis of T-ALL (d0, left), count of T-ALL cells in $\mathrm{mm}^{3}$ at day 8 (d8) and percentage of T-ALL cells in bone marrow at day 15 (d15) of T-ALL patients expressed as minimal residual disease (MRD) in IL-7 responder (circles, $n=6$ ) and IL-7 non-responder (squares, $n=10$ ) T-ALL samples are shown. Horizontal dashed line dissects prednisone (PDN) good responders (under the cut-off) and PDN poor responders (above the cutoff).

of all intracellular regulators in unstimulated T-ALL samples and we identified higher levels of $\mathrm{p}-\mathrm{RB}$ and $\mathrm{Ki}-$ 67 , both markers of cell cycle progression, in IL-7 responders compared to IL-7 non-responders (Figure 3D; Online Supplementary Figure S9). Interestingly, while both subgroups had comparable white blood cell counts at diagnosis, IL-7 responder T-ALL patients had significantly lower absolute blast cell counts at day 8 of treatment (Figure $3 \mathrm{E}$ ), the predictor of prognosis in pediatric T-ALL, ${ }^{5}$ indicating that ex vivo IL-7 responsiveness might reflect the sensitivity to prednisone therapy in vivo. In order to experimentally test the sensitivity to prednisolone, we treated either IL-7 responder (TALL-1, -3, -4, -11, -13) or IL7 non-responder (TALL-10, -12, -14, -16, -17) samples ex vivo with IL-7 and prednisolone. Cells from IL-7 responder cases significantly increased their counts upon IL-7 ad- ministration, while the cells from IL-7 non-responders did not (Online Supplementary Figure S10A). Next, we tested the ex vivo response to prednisolone in the absence or presence of IL-7. Prednisolone alone induced a significant reduction of viable cells in the culture of IL-7 responder cases but only an insignificant decrease in IL-7 non-responder cultures (Online Supplementary Figure $510 B$ and C). However, in the presence of IL-7 the effect of prednisolone in responders was mitigated to the level observed at basal condition (Online Supplementary Figure S10B).

The PI3K pathway can be inhibited in vitro by BEZ-235 in 11 of $16 \mathrm{~T}$-cell acute lymphoblastic leukemia samples

As reported previously, ${ }^{49}$ the level of basal phosphorylation of the PI3K pathway members $\mathrm{p}-4 \mathrm{E}-\mathrm{BP} 1, \mathrm{p}-\mathrm{AKT}$ and p-S6 was high in T-ALL (Figure 1). Therefore, we tested the 
inhibitory potential of the dual PI3K/mTOR inhibitor BEZ235. Since no basal activation of the PI3K pathway was observed in the residual $\mathrm{T}$ cells or $\mathrm{T}$ cells obtained from $\mathrm{PB}$ of healthy donors, we did not include any of those in the analysis. Regarding the extent of BEZ-235-induced decrease in levels of $\mathrm{p}-4 \mathrm{E}-\mathrm{BP} 1, \mathrm{p}-\mathrm{AKT}$ and $\mathrm{p}-\mathrm{S} 6$ (Figure 4A), we dissected two clusters of T-ALL patients: (i) ex vivo BEZ-235 responder T-ALL samples $(n=11)$, which showed a decreased level of $\mathrm{p}-4 \mathrm{E}-\mathrm{BP} 1$ and (ii) ex vivo BEZ-235 non-responder T-ALL samples $(n=5)$, which showed no to mild change in the level of PI3K pathway members' phosphorylation. When we compared the basal phosphorylation of p-4E-BP1, p-AKT and p-S6 in the two clusters of T-ALL, we did not find any difference (Figure 4B). However, we observed significant inactivation of 4E-BP1 and AKT only in the BEZ-235 responder T-ALL samples. Then, to

A
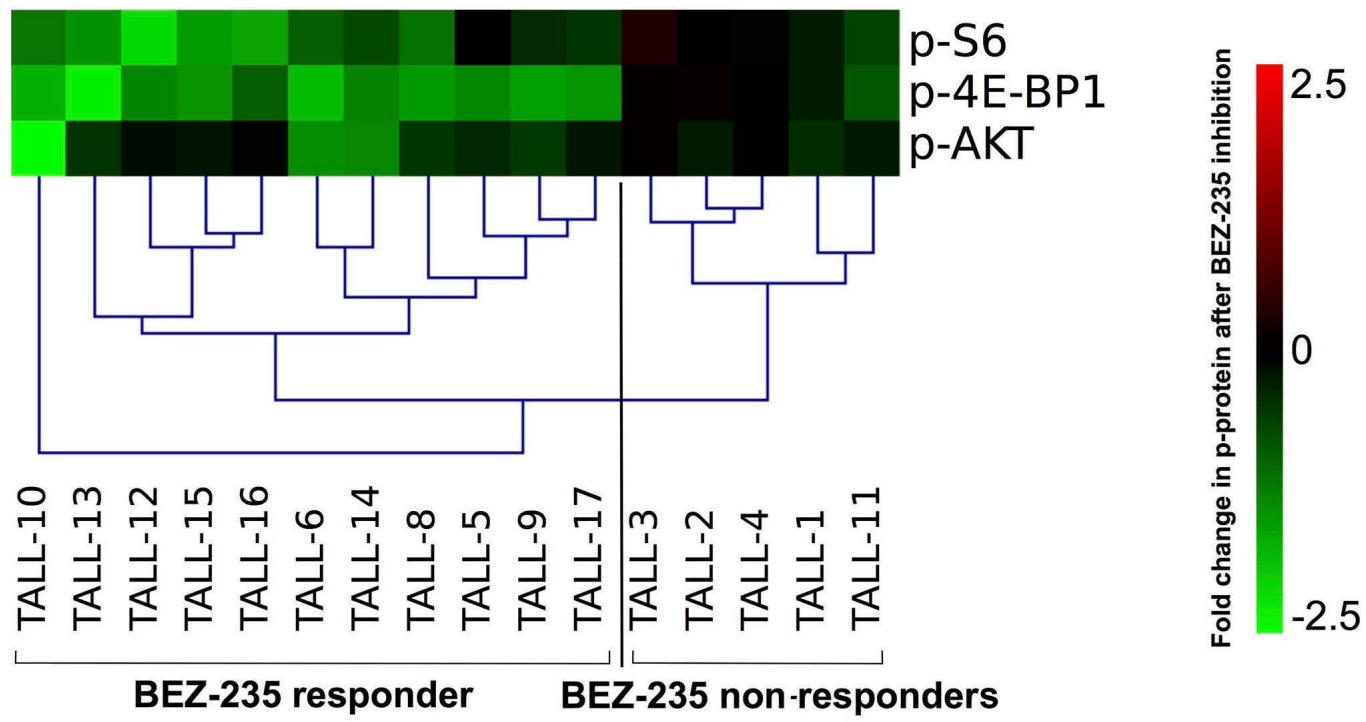

B
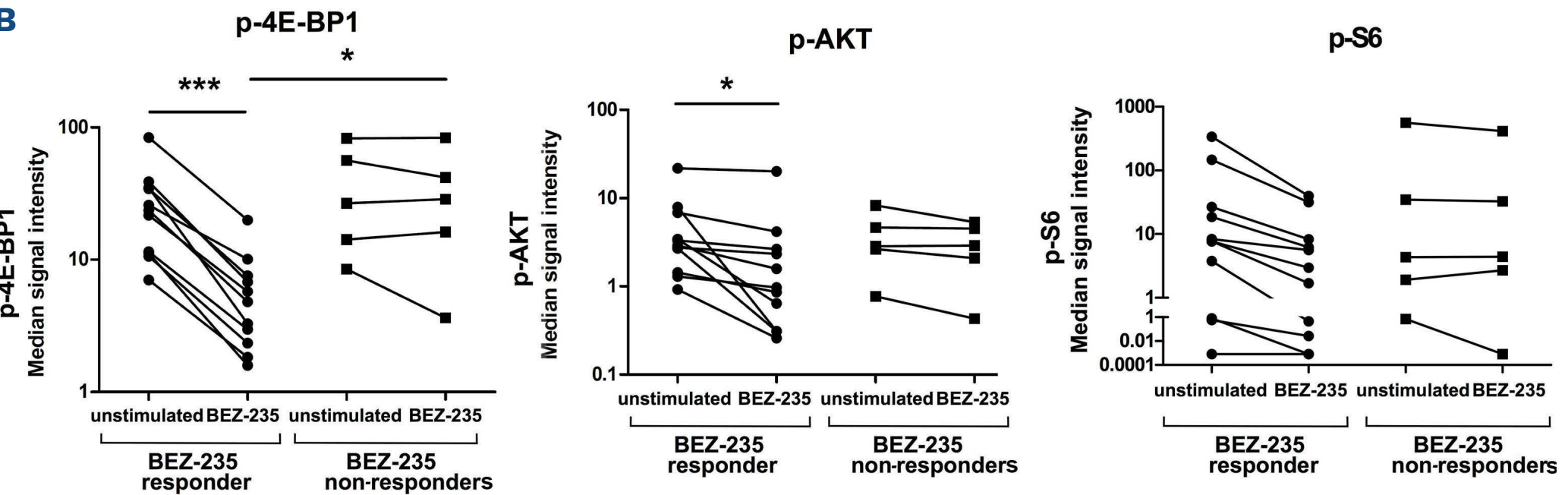

C p-LCK

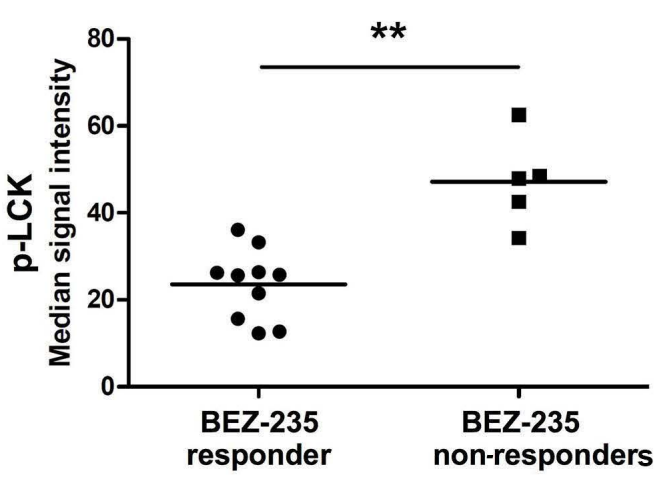

Figure 4. Response of T-cell acute lymphoblastic leukemia cells to BEZ-235 inhibition. (A) Heatmap represents fold change in protein phosphorylation of PI3K pathway members in ex vivo BEZ-235 stimulated cells compared to their unstimulated state in T-cell acute lymphoblastic leukemia (T-ALL) patients samples (in columns) obtained at diagnosis. Only gated T-ALL cells were considered in the analysis. Squares represent the fold change in phosphorylation of proteins listed in rows. Hierarchical clustering with Euclidean distance metrics and an average linkage dissects two clusters of T-ALL: BEZ-235 responder and BEZ-235 nonresponder. (B) Median intensity of p-4E-BP1, p-AKT and p-S6 in untreated and ex vivo BEZ-235 treated specimens from BEZ-235 responder T-ALL (circles, $n=11$ ) and $B E Z-235$ non-responder T-ALL (squares, $n=5$ ) samples are shown. Median intensity of $p-4 E-$ BP1 and P-AKT decreased after ex vivo BEZ-235 treatment in BEZ-235 responder T-ALL but not in BEZ-235 non-responder. (C) Median intensity of phosphorylated LCK in BEZ-235 responder T-ALL (circles, n=11) and BEZ-235 non-responder T-ALL (squares, $\mathrm{n}=5$ ) is shown. 
further investigate the features associated with non-responsiveness to BEZ-235, we compared the profiles of all the analyzed intracellular regulators (Online Supplementary Figure S11). Only the inhibitory carboxy-terminal Tyr of Lck was hyperphosphorylated in BEZ-235 non-responder TALL patients under the basal conditions (Figure 4C). Next, we wanted to investigate whether the BEZ-235 response correlates with GC responsiveness and if the addition of BEZ-235 can increase cells' sensitivity to prednisolone. We treated cells with BEZ-235 in the presence or absence of prednisolone. The decrease in viability induced by BEZ235 alone in four samples from the group considered BEZ235 responders (TALL-10, -14, -16, -17) was not significant. Furthermore, prednisolone + BEZ-235 combined treatment did not increase cells' sensitivity to GC (Online Supplementary Figure S12A). Similar results were obtained when the four samples from the group of BEZ-235 nonresponders (TALL-1, 3, 4, 11) were tested, as shown in the Online Supplementary Figure S12B.

\section{JAK-STAT pathway activation and PI3K-AKT pathway inhibition are mutually exclusive features of T-cell acute lymphoblastic leukemia}

Interestingly, we observed a high degree of mutual exclu- sivity in IL-7 and BEZ-235 responsiveness among the TALL samples: four of 16 samples (TALL-1, -3, -4, -11) were IL-7 responders but not BEZ-235 responders; in contrast, 9 of 16 samples (TALL- $6,-8,-9,-10,-12,-14,-15,-16,-17$ ) were BEZ-235 responders but IL-7 nonresponders. Two samples (TALL-5 and TALL-13) responded to both ex vivo treatments, and only one sample (TALL-2) was not responsive to any ex vivo treatment (Figure 5A; Online Supplementary Table S2), Furthermore, when we analyzed the composition of the IL-7- or BEZ-235 based clusters with regards to the in vivo response to GC (Figure $5 \mathrm{~B}$ ), we noticed that all the IL-7 responder samples were from PGR patients (Online Supplementary Table S2), while samples of BEZ-235 responders were enriched in PPR samples (6 of $9,66.6 \%$ ), suggesting a correlation between JAK-STAT or PI3K-AKT activation and the early response to treatment in vivo.

\section{Phospho-signatures and the dominance of the JAK- STAT or PI3K-AKT pathway are maintained at relapse}

Since phosphokinases are dynamic regulators of signaling networks, we investigated whether the phospho-signature present at diagnosis was maintained at relapse. Using the CуTOF panel, we analyzed five pairs of diagnostic and re-

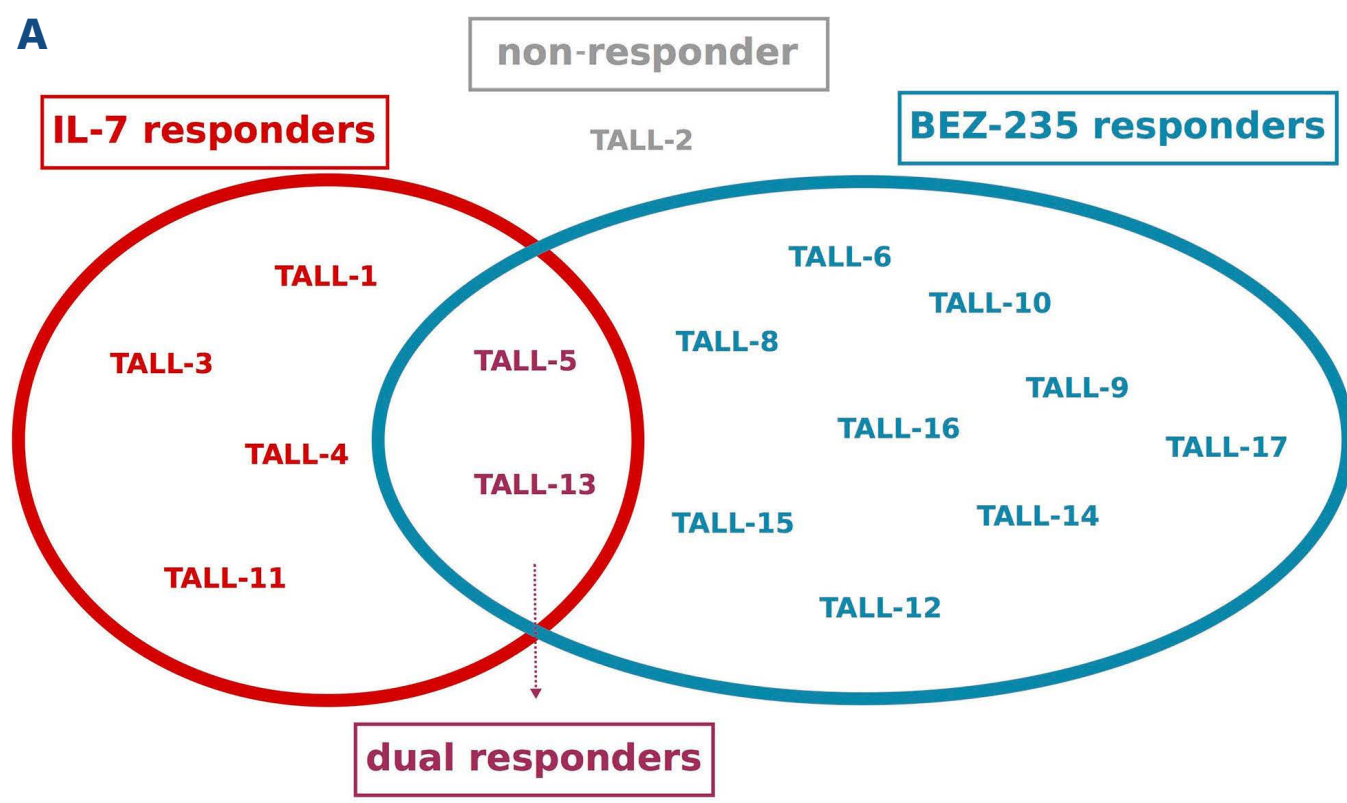

B

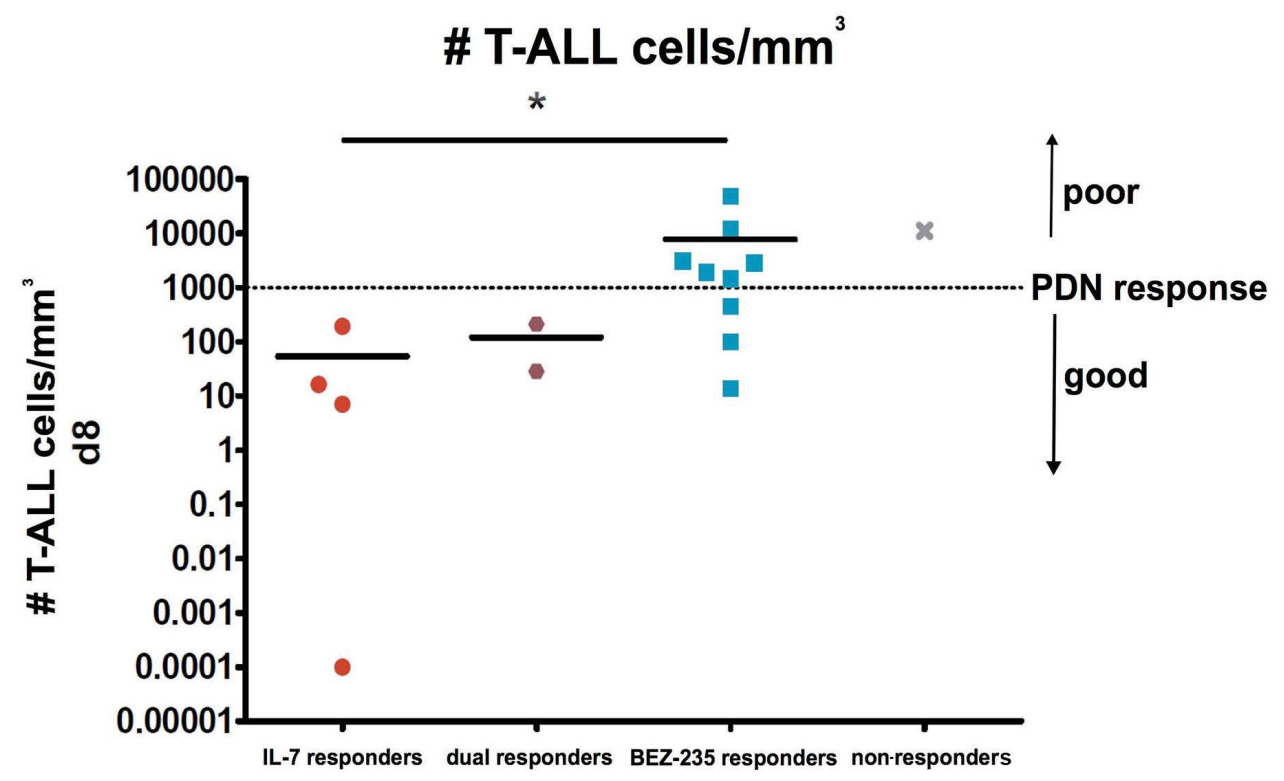

Figure 5. Dominance of JAK-STAT or PI3K-AKT pathway in pediatric T-cell acute lymphoblastic leukemia. T-cell acute lymphoblastic leukemia (T-ALL) patient samples were defined as IL-7 and/or BEZ-235 responders based on phosphorylation/dephosphorylation of particular targets as described in Figure 3 and Figure 4. TALL-2 did not respond to any of those 2 ex vivo treatment, TALL-5 and TALL-13 were defined as dual responders to IL-7 and BEZ-235 ex vivo treatment. TALL-1, TALL-3, TALL-4 and TALL-11 are IL-7 responders (and BEZ-235 non-responders). TALL-6, TALL-8, TALL9, TALL-10, TALL-12, TALL-14, TALL-15, TALL-16 and TALL-17 are BEZ-235 responders (and IL-7 non-responders). Thirteen of 16 of T-ALL patients' samples show mutual exclusivity of response. (B) Count of TALL cells $/ \mathrm{mm}^{3}$ at day 8 (d8) in IL-7 responder (red circles, $n=4)$, dual responder to IL-7 and BEZ-235 (purple hexagons, $n=2$ ), BEZ-235 responder (blue squares, $n=9$ ) and non-responder (grey cross, $n=1$ ) $T$ ALL samples are shown. Horizontal dashed line dissects prednisone (PDN) good responders (under the cut-off) and PDN poor responders (above the cut-off). 
lapsed T-ALL patient samples (TALL-1, -2, -15, -16, -17). In order to visualize phospho-signatures, Vaevictis dimensionality reduction was applied (Figure 6A). Signaling, proliferation and internal regulators (13 parameters) were considered for the projection calculation. First, we applied a gating strategy to identify the dominant and secondary clones of T-ALL cells in the diagnostic samples. Next, we applied an identical gating strategy on the relapse samples and compared the expression patterns of our markers in all paired samples (Online Supplementary Figure S13). In TALL-1, TALL-15 and TALL-16, the major diagnostic clone (accounting for $72.1 \%, 58.9 \%$ and $79.3 \%$ of cells, respectively) was the subset characterized by highly phosphorylated RB and 4E-BP1, indicating active PI3K-AKT pathway and cell cycle progression. At relapse, the major diagnostic clone was still present in TALL-1 and TALL-15, although in a reduced proportion $(24.2 \%$ and $8.93 \%$, respectively); the major clones represented at relapse $(62.3 \%$ and $67 \%$, respectively) retained intermediate levels of $p-R B$ and $p-4 E-B P 1$. In TALL-16, the dominant diagnostic clone $(79.3 \%)$ was virtually absent at relapse (0.13\%), showing a concomitant dramatic increase (84.2\%) in the clone with intermediate levels of $p-R B$ and $p-4 E-$ BP1. In TALL-2 and TALL-17, the dominant clusters at diagnosis ( $70.6 \%$ and $60.8 \%$, respectively), with intermediate levels of $\mathrm{p}-\mathrm{RB}$ and $\mathrm{p}-4 \mathrm{E}-\mathrm{BP} 1$, remained prevalent at relapse $(53.5 \%$ and $82 \%)$. In summary, we observed that in four of five paired T-ALL samples (TALL-1, -2, -15, -17), the pattern of phospho-signatures was maintained at relapse, with a redistribution of cells from clones with highly phosphorylated RB and 4E-BP1 towards clones with lower p-RB. In one sample (TALL-16), the dominant cluster (with the highest activation (evel) disappeared at relapse.

We next sought to assess whether these findings corresponded with the dominance of the JAK-STAT or PI3KAKT pathway. We performed the same ex vivo treatments applied to diagnostic samples to their relapsed counterparts. We observed that with regards to the fold change in phosphoproteins $(n=9)$ after ex vivo IL-7 stimulation, all relapsed samples were allocated to the same cluster as their diagnostic counterparts, specifically (i) ex vivo IL-7 responder T-ALL samples containing paired samples from TALL-1 and (ii) ex vivo IL-7 non-responders T-ALL samples containing paired samples from patients with TALL-2, TALL-15, TALL-16 and TALL-17 (Figure 6B). Similarly, we analyzed the BEZ-235 induced PI3K-AKT response ( $p-4 E-$ $\mathrm{BP} 1, \mathrm{p}-\mathrm{AKT}$ and $\mathrm{p}-\mathrm{S} 6$ ) in the 5 paired T-ALL samples (Figure $6 \mathrm{C}$ ); four of 5 relapsed samples were allocated to the same cluster as the diagnostic paired specimens, specifically: (i) ex vivo BEZ-235 responders TALL-15 and TALL-17; (ii) ex vivo BEZ-235 non-responders TALL-1 and TALL-2. An exception was TALL-16 in which the relapse sample shifted to the BEZ-235 non-responder cluster, while its diagnostic counterpart was originally located in the BEZ-
235 responder cluster. Notably, TALL-16 was the only sample that lost the dominant diagnostic clone (with a highly active PI3K-AKT pathway) at relapse (Figure 6A).

\section{Discussion}

In the present study we provide original evidence showing that T-ALL blasts exhibit intrapatient heterogeneity of phospho-signaling regulators that are activated at different levels within interconnected cell subsets and that this regulatory activity is generally maintained at relapse. Our study accidentally identified one patient with a subclonal PTEN mutation that demonstrated unique heterogeneity of intracellular regulators documenting that single-cell analysis by mass cytometry can tackle this type of complex patterns. Furthermore, we observed that perturbation induced by the ex vivo administration of either IL-7 or BEZ235 reveals a high degree of mutual exclusivity with respect to the responsiveness to these agents at phospho-protein level. One limitation of the present study is the low number of patients. Also, we have selected the primary samples based on the availability of frozen vials in the cell bank, the number of cells/vial and the cell viability at the time of thawing. This resulted in a final selection which does not fully reflect the classical T-ALL case distribution. In particular, our series does not contain $I L-7 R$ mutated cases and is slightly enriched in PTEN mutated patients (37.5\%) compared to the reported incidence of this subgroup in other reports such as those by $\mathrm{Pa}-$ lomero et al. ${ }^{50}$ and by Zuurbier et al. ${ }^{30}$ who documented the incidence ranging from $16 \%$ to $17 \%$ of T-ALL. In addition to the bias in genetic mutations distribution, our series is slightly enriched in PPR patients, being $43.7 \%$ compared to $34 \%$ in historical prospective series of childhood T-ALL reported by Schrappe et al. ${ }^{51}$

Mass cytometry allowed us to apply a broad panel of moAb, demonstrating for the first time the feasibility and power of this technology in the detailed characterization of signaling networks in T-ALL samples. Mass cytometry phospho-profiling was pioneered by Bodenmiller, ${ }^{36}$ who also used cellular barcoding to study the signaling dynamics and cell-to-cell communication in human blood cells. Here, we confirm and extend our previous observations by phospho-flow cytometry of hyperactivated status of T-ALL. ${ }^{38}$ We demonstrate the potential value of this technology as a tool for the identification of the dominant features of the response to IL-7 activation or BEZ-235 inhibition (here, p-STAT5 and p-4E-BP1). Importantly, barcoding of samples and gating of residual $T$ cells allowed for multiple levels of internal controls, assuring technical accuracy and proper context for interpretation. The running cost of mass cytometry technology with barcoding is comparable to conventional cytometry, while it provides the advantage of a high number of parameters, notably 


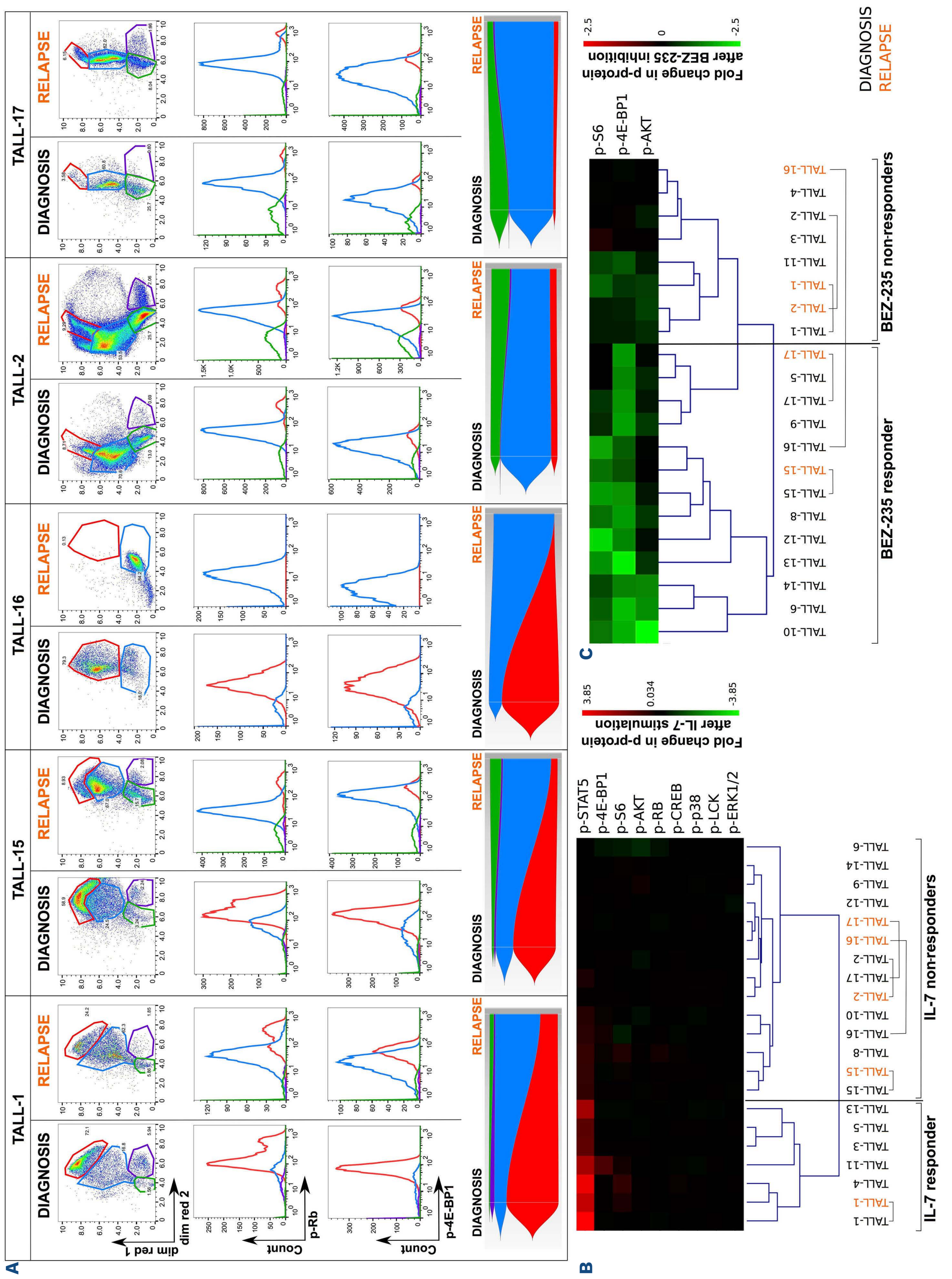


Figure 6. Single-cell and bulk analysis in T-cell acute lymphoblastic leukemia (T-ALL) cells of functional parameters reveals maintenance of phospho-signature at relapse in 4 of 5 T-ALL patients. (A) Vaevictis dimensionality reduction was applied on Tcell acute lymphoblastic leukemia (T-ALL) cells obtained from 5 pediatric T-ALL patients at diagnosis and relapse (top row). Signaling, proliferation and internal regulators (PTEN, MYC, BCL-2, p-4E-BP1, p-STAT5, p-AKT, p-p38, p-S6, p-LCK, p-CREB, p-RB, Ki-67, p-ERK1/2) were considered for the projection calculation. Two to 4 subsets of T-ALL cells were identified in each diagnostic sample. The same gating strategy was applied on the paired relapse samples. Histograms show phosphorylation of RB (middle row) and 4E-BP1 (bottom row) of particular subsets of T-ALL cells. The color of the histogram line corresponds with the color of the gate in the Vaevictis projection. The y-axis reflects the count of the cells. The redistribution of particular subsets from diagnosis to relapse is visualized using fish plot. The color of each subset corresponds with the color of the gate in the Vaevicts projection. Time points (diagnosis, relapse) are indicated on $\mathrm{x}$-axis, percentage of particular cell subset is reflected on $y$-axis. (B) Heatmap represents fold change in protein phosphorylation in ex vivo IL-7 stimulated cells compared to unstimulated state, samples were obtained from T-ALL patients (in columns) at diagnosis (black label) or relapse (orange label). Only T-ALL cells from the diagnostic samples are included in the analysis. Paired diagnostic and relapse samples are connected. Heat in squares represents the fold change in phosphorylation of proteins listed in rows. Hierarchical clustering with Euclidean distance metrics and an average linkage dissects two clusters of T-ALL samples (separated with horizontal line): IL-7 responder and IL-7 non-responder. (C) Heatmap represents fold change in protein phosphorylation of PI3K pathway members in ex vivo BEZ-235 stimulated cells compared to unstimulated state, samples were obtained from T-ALL patients (in columns) at diagnosis (black label) or relapse (orange label). Only T-ALL cells from the diagnostic samples are included in the analysis. Paired diagnostic and relapse samples are connected. Heat in squares represents the fold change in phosphorylation of proteins listed in rows. Hierarchical clustering with Euclidean distance metrics and an average linkage was used. The 2 clusters of samples (separated with horizontal line) were dissected manually according to Figure 4: BEZ-235 responder and BEZ-235 non-responder.

phosphoproteins, that can be reliably measured. Our workflow overcomes some limitations of mass cytometry (high equipment cost and demanding reagent stock production) by shipping fixed samples after in vitro treatment to a collaborator.

The Vaevictis projection shows recurrent patterns in patients with particular mutations. Notably, PTENmut cells displayed high MYC expression. MYC is a master transcription factor regulating several critical cell functions, such as metabolism, proliferation and survival, ${ }^{52}$ and it functions as a potent oncogene in a large number of cancers. ${ }^{53}$ In line with our findings, Bonnet et al. ${ }^{54}$ showed that modulation of MYC protein was maintained through downregulation of PTEN via the PI3K-AKT axis in T-ALL. This explains the significant inverse correlation between MYC and PTEN expression. Furthermore we analyzed the correlation between NOTCH1, PTEN status and MYC levels. We observed that MYC was low in all PTENwt cases regardless of NOTCH1 status, while all the PTENmut cases displayed high MYC in line with Bonnet et al. ${ }^{54}$ These authors showed that high MYC expression levels can be observed in the absence of (known) NOTCH1 and/or FBXW7 mutations and that modulation of MYC protein can be observed in the presence of downregulation of PTEN as a major alternative pathway of MYC activation in T-ALL independently from NOTCH1 mutations.

$\mathrm{GC}$ resistance remains a major challenge in the treatment of pediatric ALL, and although several mechanisms have been suggested, ${ }^{6,55,56}$ our understanding of the molecular basis remains incomplete.

We found that all IL-7 responding samples were from patients with a good response to GC in vivo whereas all BEZ235 responding (but IL-7 non-responsive) samples were from patients with poor response to GC in vivo. However, due to the limited number of patients studied we cannot state that IL-7R pathway activation in vitro reflects the sensitivity to PDN therapy in vivo. Nevertheless, our preliminary observations are also supported by GC cytotoxicity experiments that assessed ex vivo prednisolone response in T-ALL primary cells in the absence or presence of IL-7.

Firstly, we observed that in samples that responded to IL7 stimulation by increased phosphorylation of STAT5 the number of viable cells significantly increased upon ex vivo administration of IL-7. In contrast, the IL-7 non-responsive samples did not show any significant increase. We then exposed both kinds of samples to prednisolone in the absence or presence of IL-7. In the absence of IL-7, we observed a significant reduction in the number of viable cells, whereas when combined, IL-7 and prednisolone mutually canceled out their effects. Similar investigations were carried out by Delgado-Martin et $a l .^{57}$ who assessed p-STAT5 responsiveness to IL-7 with regards to GC sensitivity, and he found that the responder subsets displayed resistance to GC-induced death while the IL-7 non-responder subset was mostly GC sensitive. However these authors did not formally assess the pro-survival effect of IL-7 alone, but only as a protective factor from chemotherapy-induced death.

In our series of primary T-ALL samples, the IL-7 response was assessed as both p-STAT5 response and the pro-survival response. These characteristics correlated with good GC response in vivo. Notably, the IL-7 responder cells in our series displayed a significantly higher expression of both Ki-67 and p-RB indicating a higher proliferative functional state as compared to IL-7 non-responder blasts. This is consistent with a more effective action of GC and other cytotoxic drugs on highly proliferating cells. ${ }^{58,59}$

Furthermore, we assessed whether the observed fold change in phospho-proteins after BEZ-235 treatment cor- 
related with drug sensitivity, and if its addition could increase cell sensitivity to GC. Indeed, BEZ-235 treatment did not increase the cell sensitivity to prednisolone in the four BEZ-235 phospho-responder samples. Hall and collaborators $^{60}$ examined the cytotoxic activity of BEZ-235 and dexamethasone, as single agents and in combination in both T-ALL cell lines and primary T-ALL samples. BEZ235 alone did not show any significant activity, in line with our data. However, these authors observed a synergistic cytotoxic effect of BEZ-235 and dexamethasone in most T-ALL cell lines tested, and in primary T-ALL lymphoblast. $\mathrm{Li}$ and colleagues ${ }^{19}$ studied a large cohort of primary TALL samples and demonstrated that genes involved in the IL7R-RAS-MAPK-AKT signaling pathway were significantly associated with steroid resistance and poor clinical outcome. They subsequently evaluated the drug synergism between PDN and several agents including a PI3K-AKTmTOR inhibitor in 11 primary T-ALL samples collected at diagnosis and they observed an enhanced steroid response in most samples in line with the data by Hall et $a l .{ }^{60}$ We tested the synergism between prednisolone and BEZ-235 in only four patients and we did not perform any extended screening of the mutational state of the IL-7R pathway, thus our observation of the lack of additive effect of BEZ-235 and PDN activity should be taken with caution.

Recently, Liu et al. ${ }^{22}$ performed an integrated genomic analysis of a large cohort of T-ALL patients and identified ten functional pathways recurrently mutated in T-ALL, including the PI3K-AKT-mTOR and JAK-STAT pathways, in $29 \%$ and $25 \%$ of cases, respectively. Mutual exclusivity was observed between the PI3K-AKT pathway and JAKSTAT or Ras pathway alterations. Our data are in line with this hypothesis at least at the phospho-proteomic level as we found two subsets of patients with opposite response to IL-7 and to BEZ-235.

Notably, the signature associated with either IL-7 or BEZ235 responsiveness was maintained at relapse, with the exception of a single case in which the loss of the highly activated dominant clone was observed. In this regard the importance of the conservation of the functional dominance suggests that functional studies at an early stage (i.e., at diagnosis) may be of relevance for treatment of relapse. Therefore cases of refractory T-ALL patients warrant further in-depth studies.

We are well aware that any clinical correlation of our biology-oriented study in a limited non-representative series of childhood T-ALL (for example no IL-7R mutated patients are included) should be corroborated in further extensive investigations, nevertheless we do believe that our data should be considered in view of a better understanding of the mechanisms of GC resistance in childhood T-ALL.

In summary, we demonstrated the translational potential of the mass cytometry-based T-ALL profiling at the single-cell level. Further, our analysis allowed us to investigate the heterogeneity within a single T-ALL sample, revealing a rare case of subclonal PTEN mutation ${ }^{28}$.

Because of this advanced approach, we were able to identify distinct functional clusters of IL-7 and BEZ-235 responsive T-ALL cells with different sensitivity to GC, which can persist at relapse. Collectively, our observations can contribute to a better understanding of the complex signaling network governing T-ALL behavior and its correlation with the response to therapy.

\section{Disclosures}

No conflicts of interest to disclose.

\section{Contributions}

$D K$ and $C B$ performed the experiments, analyzed the data, wrote the manuscript; KR performed the experiments; ARS performed the conjugations experiments; HEM designed and performed conjugations experiments; MP performed part of the mutation screening; ABif and Abio revised the manuscript; TK and GG designed the research, analyzed the data and wrote themanuscript. All authors have read and approved the final submitted version of the manuscript

\section{Acknowledgments}

We wish to thank to Ondrej Hrusak for fruitful discussions and to Daniel Thürner and Pavel Semerak for superb handling of the Helios instrument.

\section{Funding}

This project was supported by the Fondazione Alessandro Maria Zancan ONLUS "Grande Ale ONLUS", Fondazione M. Tettamanti De Marchi and NU20-05-00282 from the Ministry of Health, Czech Republic. It was also partially funded by the following grants: AIRC IG 2017 ref. id 20564, AIRC $5 \times 1000$ ref. id 21147 to $A B, A I R C$ Accelerator Award 2018 ref. id 22791.

\section{References}

1. Pui $\mathrm{CH}$. Acute lymphoblastic leukemia: introduction. Semin Hematol. 2009;46(1):1-2.

2. Girardi T, Vicente C, Cools J, Keersmaecker K De. The genetics and molecular biology of T-ALL. Blood. 2017;129(9):1113-1123.

3. Klumper E, Pieters R, Veerman A, et al. In vitro cellular drug resistance in children with relapsed/refractory acute 
lymphoblastic leukemia. Blood. 1995;86(10):3861-3868.

4. Gao J, Liu WJ. Prognostic value of the response to prednisone for children with acute lymphoblastic leukemia: a metaanalysis. Eur Rev Med Pharmacol Sci. 2018;22(22):7858-7866.

5. Lauten M, Moricke A, Beier R, et al. Prediction of outcome by early bone marrow response in childhood acute lymphoblastic leukemia treated in the ALL-BFM 95 trial: differential effects in precursor B-cell and T-cell leukemia. Haematologica. 2012;97(7):1048-1056.

6. Follini E, Marchesini M, Roti G. Strategies to overcome resistance mechanisms in T-cell acute lymphoblastic leukemia. Int J Mol Sci. 2019;20(12):3021.

7. Scheijen B. Molecular mechanisms contributing to glucocorticoid resistance in lymphoid malignancies. Cancer Drug Resist. 2019;2:647-664.

8. De Smedt R, Morscio J, Goossens S, Van Vlierberghe P. Targeting steroid resistance in T-cell acute lymphoblastic leukemia. Blood Rev. 2019;38:100591.

9. Rich BE, Campos-Torres J, Tepper RI, Moreadith RW, Leder P. Cutaneous lymphoproliferation and lymphomas in interleukin 7 transgenic mice. J Exp Med. 1993;177(2):305-316.

10. Lindqvist CM, Lundmark A, Nordlund J, et al. Deep targeted sequencing in pediatric acute lymphoblastic leukemia unveils distinct mutational patterns between genetic subtypes and novel relapse-associated genes. Oncotarget. 2016;7(39):6407164088.

11. Abraham N, Ma MC, Snow JW, Miners MJ, Herndier BG, Goldsmith MA. Haploinsufficiency identifies STAT5 as a modifier of IL-7-induced lymphomas. Oncogene. 2005;24(33):5252-5257.

12. Silva A, Laranjeira ABA, Martins LR, et al. IL-7 contributes to the progression of human T-cell acute lymphoblastic leukemias. Cancer Res. 2011;71(14):4780-4789.

13. Dibirdik I, Langlie M, Ledbetter J, et al. Engagement of interleukin-7 receptor stimulates tyrosine phosphorylation, phosphoinositide turnover, and clonal proliferation of human Tlineage acute lymphoblastic leukemia cells. Blood. 1991;78(3):564-570.

14. Treanor LM, Zhou S, Janke L, et al. Interleukin-7 receptor mutants initiate early $T$ cell precursor leukemia in murine thymocyte progenitors with multipotent potential. J Exp Med. 2014;211(4):701-713.

15. Ribeiro D, Melão A, van Boxtel R, et al. STAT5 is essential for IL7-mediated viability, growth, and proliferation of T-cell acute lymphoblastic leukemia cells. Blood Adv. 2018;2(17):2199-2213.

16. Shochat C, Tal N, Bandapalli OR, et al. Gain-of-function mutations in interleukin-7 receptor- $\alpha$ (IL7R) in childhood acute lymphoblastic leukemias. J Exp Med. 2011;208(5):901-908.

17. Zenatti PP, Ribeiro D, Li W, et al. Oncogenic IL7R gain-offunction mutations in childhood T-cell acute lymphoblastic leukemia. Nat Genet. 2011;43(10):932-939.

18. Canté-Barrett K, Spijkers-Hagelstein JAP, Buijs-Gladdines JGCAM, et al. MEK and PI3K-AKT inhibitors synergistically block activated IL7 receptor signaling in T-cell acute lymphoblastic leukemia. Leukemia. 2016;30(9):1832-1843.

19. Li Y, Buijs-Gladdines JGCAM, Canté-Barrett K, et al. IL-7 receptor mutations and steroid resistance in pediatric $T$ cell acute lymphoblastic leukemia: a genome sequencing study. PLoS Med. 2016;13(12):e1002200.

20. Oliveira ML, Akkapeddi P, Alcobia I, et al. From the outside, from within: biological and therapeutic relevance of signal transduction in T-cell acute lymphoblastic leukemia. Cell Signal. 2017;38:10-25.

21. Kim R, Boissel N, Touzart A, et al. Adult T-cell acute lymphoblastic leukemias with IL7R pathway mutations are slow-responders who do not benefit from allogeneic stem-cell transplantation. Leukemia. 2020;34(7):1730-1740.

22. Liu Y, Easton J, Shao Y, et al. The genomic landscape of pediatric and young adult T-lineage acute lymphoblastic leukemia. Nat Genet. 2017;49(8):1211-1218.

23. Goossens S, Radaelli E, Blanchet $O$, et al. ZEB2 drives immature T-cell lymphoblastic leukaemia development via enhanced tumour-initiating potential and IL-7 receptor signalling. Nat Commun. 2015;6(1):5794.

24. Zhang J, Ding L, Holmfeldt L, et al. The genetic basis of early Tcell precursor acute lymphoblastic leukaemia. Nature. 2012;481(7380):157-163.

25. Vicente C, Schwab C, Broux M, et al. Targeted sequencing identifies associations between IL7R-JAK mutations and epigenetic modulators in T-cell acute lymphoblastic leukemia. Haematologica. 2015;100(10):1301-1310.

26. Meyer LK, Huang BJ, Delgado-Martin C, et al. Glucocorticoids paradoxically facilitate steroid resistance in $\mathrm{T}$ cell acute lymphoblastic leukemias and thymocytes. J Clin Invest. 2020;130(2):863-876.

27. Okkenhaug K, Vanhaesebroeck B. PI3K in lymphocyte development, differentiation and activation. Nat Rev Immunol. 2003;3(4):317-330.

28. Mendes RD, Sarmento LM, Canté-Barrett K, et al. PTEN microdeletions in T-cell acute lymphoblastic leukemia are caused by illegitimate RAG-mediated recombination events. Blood. 2014;124(4):567-578.

29. Milella M, Falcone I, Conciatori F, et al. PTEN: multiple functions in human malignant tumors. Front Oncol. 2015;5:24.

30. Zuurbier L, Petricoin EF, Vuerhard MJ, et al. The significance of PTEN and AKT aberrations in pediatric T-cell acute lymphoblastic leukemia. Haematologica. 2012;97(9):1405-1413.

31. Homminga I, Pieters R, Langerak AW, et al. Integrated transcript and genome analyses reveal NKX2-1 and MEF2C as potential oncogenes in T cell acute lymphoblastic leukemia. Cancer Cell. 2011;19(4):484-497.

32. Ferrando AA, Neuberg DS, Staunton J, et al. Gene expression signatures define novel oncogenic pathways in $T$ cell acute lymphoblastic leukemia. Cancer Cell. 2002;1(1):75-87.

33. Soulier J, Clappier E, Cayuela JM, et al. HOXA genes are included in genetic and biologic networks defining human acute T-cell leukemia (T-ALL). Blood. 2005;106(1):274-286.

34. Bhatla T, Jones CL, Meyer JA, Vitanza NA, Raetz EA, Carroll WL. The biology of relapsed acute lymphoblastic leukemia. J Pediatr Hematol Oncol. 2014;36(6):413-418.

35. Loosveld M, Castellano R, Gon S, et al. Therapeutic targeting of C-Myc in T-cell acute lymphoblastic leukemia (T-ALL). Oncotarget. 2014;5(10):3168-3172.

36. Bodenmiller B, Zunder ER, Finck R, et al. Multiplexed mass cytometry profiling of cellular states perturbed by smallmolecule regulators. Nat Biotechnol. 2012;30(9):858-867.

37. Bendall SC, Simonds EF, Qiu P, et al. Single-cell mass cytometry of differential immune and drug responses across a human hematopoietic continuum. Science. 2011;332(6030):687-696.

38. Bonaccorso P, Bugarin C, Buracchi C, et al. Single-cell profiling of pediatric T-cell acute lymphoblastic leukemia: impact of PTEN exon 7 mutation on PI3K/Akt and JAK-STAT signaling pathways. Cytometry B Clin Cytom. 2020;98(6):491-503.

39. Dworzak MN, Buldini B, Gaipa G, et al. AlEOP-BFM consensus guidelines 2016 for flow cytometric immunophenotyping of pediatric acute lymphoblastic leukemia. Cytometry B Clin Cytom. 2018;94(1):82-93.

40. Watson M, Chow S, Barsyte D, et al. The study of epigenetic mechanisms based on the analysis of histone modification 
patterns by flow cytoametry. Cytometry A. 2014;85(1):78-87.

41. Mei HE, Leipold MD, Maecker HT. Platinum-conjugated antibodies for application in mass cytometry. Cytometry A. 2016;89(3):292-300.

42. Mei HE, Leipold MD, Schulz AR, Chester C, Maecker HT. Barcoding of live human peripheral blood mononuclear cells for multiplexed mass cytometry. J Immunol. 2015;194(4):2022-2031.

43. Schulz AR, Baumgart S, Schulze J, Urbicht M, Grützkau A, Mei HE. Stabilizing antibody cocktails for mass cytometry. Cytometry A. 2019;95(8):910-916.

44. Chevrier S, Crowell HL, Zanotelli VRT, Engler S, Robinson MD, Bodenmiller B. Compensation of signal spillover in suspension and imaging mass cytometry. Cell Syst. 2018;6(5):612-620.

45. Saeed Al, Sharov V, White J, et al. TM4: a free, open-source system for microarray data management and analysis. Biotechniques. 2003;34(2):374-378.

46. Szubert B, Cole JE, Monaco C, Drozdov I. Structure-preserving visualisation of high dimensional single-cell datasets. Sci Rep. 2019;9(1):8914.

47. Ding J, Condon A, Shah SP. Interpretable dimensionality reduction of single cell transcriptome data with deep generative models. Nat Commun. 2018;9(1):2002.

48. Sulis ML, Williams O, Palomero T, et al. NOTCH1 extracellular juxtamembrane expansion mutations in T-ALL. Blood. 2008;112(3):733-740.

49. Silva A, Yunes JA, Cardoso BA, et al. PTEN posttranslational inactivation and hyperactivation of the PI3K/Akt pathway sustain primary $\mathrm{T}$ cell leukemia viability. J Clin Invest. 2008;118(11):3762-3774.

50. Palomero T, Sulis ML, Cortina M, et al. Mutational loss of PTEN induces resistance to NOTCH1 inhibition in T-cell leukemia. Nat Med. 2007;13(10):1203-1210.

51. Schrappe M, Valsecchi MG, Bartram CR, et al. Late MRD response determines relapse risk overall and in subsets of childhood T-cell ALL: results of the AIEOP-BFM-ALL 2000 study. Blood. 2011;118(8):2077-2084.

52. Dang C V., O’Donnell KA, Zeller KI, Nguyen T, Osthus RC, Li F.
The c-Myc target gene network. Semin Cancer Biol. 2006;16(4):253-264.

53. Meyer N, Penn LZ. Reflecting on 25 years with MYC. Nat Rev Cancer. 2008;8(12):976-990.

54. Bonnet M, Loosveld M, Montpellier B, et al. Posttranscriptional deregulation of MYC via PTEN constitutes a major alternative pathway of MYC activation in T-cell acute lymphoblastic leukemia. Blood. 2011;117(24):6650-6659.

55. Piovan E, Yu J, Tosello V, et al. Direct reversal of glucocorticoid resistance by AKT inhibition in acute lymphoblastic leukemia. Cancer Cell. 2013;24(6):766-776.

56. Serafin V, Capuzzo G, Milani G, et al. Glucocorticoid resistance is reverted by LCK inhibition in pediatric T-cell acute lymphoblastic leukemia. Blood. 2017;130(25):2750-2761.

57. Delgado-Martin C, Meyer LK, Huang BJ, et al. JAK/STAT pathway inhibition overcomes IL7-induced glucocorticoid resistance in a subset of human T-cell acute lymphoblastic leukemias. Leukemia. 2017;31(12):2568-2576.

58. Ebinger S, Özdemir EZ, Ziegenhain C, et al. Characterization of rare, dormant, and therapy-resistant cells in acute lymphoblastic leukemia. Cancer Cell. 2016;30(6):849-862.

59. Martelli AM, Lonetti A, Buontempo F, et al. Targeting signaling pathways in T-cell acute lymphoblastic leukemia initiating cells. Adv Biol Regul. 2014;56:6-21.

60. Hall CP, Reynolds CP, Kang MH. Modulation of glucocorticoid resistance in pediatric $T$-cell acute lymphoblastic leukemia by increasing BIM expression with the PI3K/MTOR inhibitor BEZ235. Clin Cancer Res. 2016;22(3):621-632.

61. Coustan-Smith E, Mullighan CG, Onciu M, et al. Early T-cell precursor leukaemia: a subtype of very high-risk acute lymphoblastic leukaemia indentified in two independent cohorts. Lancet Oncol. 2009;10(2):147-156.

62. Basso G, Veltroni M, Valsecchi MG, et al. Risk of relapse of childhood acute lymphoblastic leukemia is predicted by flow cytometric measurement of residual disease on day 15 bone marrow. J Clin Oncol. 2009;27(31):5168-5174. 